University of Wollongong

Research Online

Faculty of Social Sciences - Papers (Archive) Faculty of Arts, Social Sciences \& Humanities

2014

Preferred EEG brain states at stimulus onset in a fixed interstimulus interval equiprobable auditory Go/NoGo task: A definitive study

Robert Barry

University of Wollongong, rbarry@uow.edu.au

Frances M. De Blasio

University of Wollongong, francesd@uow.edu.au

Vilfredo De Pascalis

University of Rome

Diana Karamacoska

University of Wollongong, dk744@uowmail.edu.au

Follow this and additional works at: https://ro.uow.edu.au/sspapers

Part of the Education Commons, and the Social and Behavioral Sciences Commons

Research Online is the open access institutional repository for the University of Wollongong. For further information contact the UOW Library: research-pubs@uow.edu.au 


\title{
Preferred EEG brain states at stimulus onset in a fixed interstimulus interval equiprobable auditory Go/NoGo task: A definitive study
}

\begin{abstract}
This study examined the occurrence of preferred EEG phase states at stimulus onset in an equiprobable auditory Go/NoGo task with a fixed interstimulus interval, and their effects on the resultant event-related potentials (ERPs). We used a sliding short-time FFT decomposition of the EEG at $\mathrm{Cz}$ for each trial to assess prestimulus EEG activity in the delta, theta, alpha and beta bands. We determined the phase of each 2. $\mathrm{Hz}$ narrow-band contributing to these four broad bands at 125 . ms before each stimulus onset, and for the first time, avoided contamination from poststimulus EEG activity. This phase value was extrapolated 125. ms to obtain the phase at stimulus onset, combined into the broad-band phase, and used to sort trials into four phase groups for each of the four broad bands. For each band, ERPs were derived for each phase from the raw EEG activity at 19 sites. Data sets from each band were separately decomposed using temporal Principal Components Analyses with unrestricted VARIMAX rotation to extract N1-1, PN, P2, P3, SW and LP components. Each component was analysed as a function of EEG phase at stimulus onset in the context of a simple conceptualisation of orthogonal phase effects (cortical negativity vs. positivity, negative driving vs. positive driving, waxing vs. waning). The predicted nonrandom occurrence of phase-defined brain states was confirmed. The preferred states of negativity, negative driving, and waxing were each associated with more efficient stimulus processing, as reflected in amplitude differences of the components. The present results confirm the existence of preferred brain states and their impact on the efficiency of brain dynamics in perceptual and cognitive processing.
\end{abstract}

\section{Keywords}

preferred, eeg, fixed, study, definitive, interstimulus, states, interval, stimulus, equiprobable, task, nogo, auditory, brain, go, onset

\section{Disciplines}

Education | Social and Behavioral Sciences

\section{Publication Details}

Barry, R. J., De Blasio, F. M., De Pascalis, V. \& Karamacoska, D. (2014). Preferred EEG brain states at stimulus onset in a fixed interstimulus interval equiprobable auditory Go/NoGo task: A definitive study. International Journal of Psychophysiology, 94 (1), 42-58. 
Preferred EEG brain states at stimulus onset in a fixed interstimulus interval equiprobable auditory Go/NoGo task: A definitive study

\section{Robert J. Barry ${ }^{*}, 1$, Frances M. De Blasio ${ }^{1}$, Vilfredo De Pascalis ${ }^{2}$, Diana Karamacoska ${ }^{1}$}

${ }^{1}$ Centre for Psychophysics, Psychophysiology, and Psychopharmacology, Brain \& Behaviour Research Institute, and School of Psychology, University of Wollongong, Northfields Avenue, Wollongong NSW 2522, Australia ${ }^{2}$ Department of Psychology, University of Rome “La Sapienza”, Rome, Italy

\section{Notes to type-setter}

1. "Brain \& Behaviour Research Institute” is a registered name and should not be changed in any way.

2. Italics (e.g., “negativity”) are used to indicate defined EEG phases, not as emphasis, and should be retained unchanged.

*Corresponding author:

Tel./Fax: +61 242214421.

E-mail address: robert_barry@uow.edu.au (R.J. Barry)

Address: School of Psychology, University of Wollongong, Wollongong NSW 2522, Australia. 


\begin{abstract}
This study examined the occurrence of preferred EEG phase states at stimulus onset in an equiprobable auditory Go/NoGo task with a fixed interstimulus interval, and their effects on the resultant event-related potentials (ERPs). We used a sliding short-time FFT decomposition of the EEG at Cz for each trial to assess prestimulus EEG activity in the delta, theta, alpha and beta bands. We determined the phase of each $2 \mathrm{~Hz}$ narrow-band contributing to these four broad bands at 125 ms before each stimulus onset, and for the first time, avoided contamination from postimulus EEG activity. This phase value was extrapolated $125 \mathrm{~ms}$ to obtain the phase at stimulus onset, combined into the broad-band phase, and used to sort trials into four phase groups for each of the four broad bands. For each band, ERPs were derived for each phase from the raw EEG activity at 19 sites. Data sets from each band were separately decomposed using temporal Principal Components Analyses with unrestricted Varimax rotation to extract N1-1, PN, P2, P3, SW and LP components. Each component was analysed as a function of EEG phase at stimulus onset in the context of a simple conceptualisation of orthogonal phase effects (cortical negativity vs. positivity, negative driving vs. positive driving, waxing vs. waning). The predicted nonrandom occurrence of phase-defined brain states was confirmed. The preferred states of negativity, negative driving, and waxing were each associated with more efficient stimulus processing, as reflected in amplitude differences of the components. The present results confirm the existence of preferred brain states and their impact on the efficiency of brain dynamics in perceptual and cognitive processing.
\end{abstract}

Keywords: Brain dynamics; Preferred phase states; Event-related potentials; Orthogonal phase effects; Phase synchronisation; Auditory Go/NoGo task; Principal Components Analysis 


\section{Introduction}

It is now well established that the brain's processing of each event in cognitive/perceptual tasks depends on the current amplitude and phase of the electroencephalogram (EEG) activity at stimulus onset (Barry, 2009; Başar, 1980; Makeig et al., 2004). In this context, an important line of brain-dynamics research focused on the phase of the ongoing EEG began with Başar and Stampfer (1985). They found that stimuli repeatedly presented at a fixed interval produced what they called a "preferred phase angle” in the ongoing EEG at stimulus onset, particularly in the delta and alpha frequency bands. That is, rather than the phase at stimulus onset being random, it was found that cortical negativity preferentially occurred. The occurrence of such preferred phase states was intriguing when considered with earlier reports that presenting stimuli at negative peaks of the alpha cycle produced shorter reaction times (Callaway \& Yeager, 1960; Trimble \& Potts, 1975). Together these suggest that Başar and Stampfer's (1985) phase reordering may be of general importance in perceptual/cognitive functioning. In relatable research, Rémond and Lesèvre (1967) had reported that stimuli presented at the negative peak of alpha activity produced visual-evoked ERP enhancements, which they considered to be an indirect effect of a non-specific mechanism increasing the state of alertness. Similar dynamic phase adjustments had also been noted previously (Başar et al., 1984; Pleydell-Pearce, 1994; Rockstroh et al., 1989), and this research collectively provided the impetus for our interest in brain dynamics, particularly in relation to the phase-reset model of ERP genesis (e.g., Barry, 2009).

Rather than working directly with phase angles in the EEG context, Barry et al. (2003) introduced a set of more-intuitive phase groupings, using the physical dimensions of phase based on the quartile divisions of a negative-up sine wave shown on the left in Figure 1. Cortical negativity/positivity compares the effects of phase divisions $(A+B)$ versus $(C+D)$, the preferred phases explored by Başar and Stampfer (1985) and covering the prior alpha peak/trough studies. Negative/positive driving, comparing $(\mathrm{A}+\mathrm{D})$ versus $(\mathrm{B}+\mathrm{C})$, assesses the change in cortical negativity (increasing vs. decreasing), accommodating other ERP effects reported for stimuli presented at the positive-going zero crossing of alpha activity (Jansen \& Brandt, 1991; Rémond \& Lesèvre, 1967). A third dimension, waxing $(\mathrm{A}+\mathrm{C})$ versus waning $(\mathrm{B}+\mathrm{D})$, was introduced by Barry et al. (2004) in a study of alpha phase effects, and refers to the change in amplitude (increasing vs. decreasing), regardless of its polarity. These three dimensions represent a set of orthogonal (statistically independent) comparisons among the means of the four quartiles of phase activity defined in Figure 1. In order to assist communication throughout this paper, we use italics to label one extreme of each of these orthogonal dimensions of EEG phase: negativity, negative driving, and waxing.

Fig. 1 about here 
Over the years our research group has systematically explored the preferential phase phenomenon and its effects. Our initial studies examined effects on ERPs in auditory oddball tasks. As subdividing in terms of phase at stimulus onset requires many trials for adequate numbers to form average ERPs in each phase, we could examine phase effects only in the ERPs to the more-prevalent standards. In our first study we used a fixed interstimulus interval (ISI) auditory oddball task with $15 \%$ target probability; targets required a button-press response. Narrow (1 Hz wide) EEG bands were found to be dynamically adjusted to provide “preferred brain states” at stimulus onset (Barry et al., 2003), compatible with Başar and Stampfer's (1985) earlier description of band effects. We found preferential occurrence, at up to double the rate expected by chance, for some of the phase states (e.g. negativity c.f. positivity) in specific frequencies (e.g., at 4, 5, and $6 \mathrm{~Hz}$ ). Some of these results bridged the borders of the traditional EEG bands (e.g., negative driving at 6, 7, 8, and $9 \mathrm{~Hz}$ ), encouraging us to continue with our narrow-band approach. Importantly, these preferred phase states were also linked to large effects in the ERPs to standards, demonstrating their impact on the cortical processing involved. For example, N1 amplitude was substantially enhanced in negative driving phases at 1, 2, 3, 4, and $5 \mathrm{~Hz}$.

Thus we continued with exploration of preferred narrow-band EEG phase effects in the auditory oddball. Barry et al. (2006, 2007) reported preliminary studies using data on standards from 1. a passive paradigm, comparing groups of subjects with low- vs. high-intensity standards, and 2. the passive low-intensity standards group with data from a group that had to button-press in response to the deviants in the identical auditory sequence. The parameters were broadly comparable to those used by Barry et al. (2003), although the fixed ISI was changed to a slightly-varying ISI. Importantly, and as predicted, the occurrence of preferred brain states was reduced by this change in ISI. The change in task produced differences in prestimulus preparatory processes, preferred brain states, and ERPs to the standards. Preferred brain states ( $20 \%$ more frequent than expected by chance) again produced more-efficient processing. These exploratory oddball studies were then extended with additional subjects to form a full factorial design (Barry et al., 2009), with groups in both passive and active oddball tasks with high and low intensity standards. This allowed testing of interactions between task and stimulus intensity. That integrative study confirmed the existence of preferred phase-defined brain states, obtained clear phase effects in each ERP component measured, and confirmed that the preferred brain states observed were functionally effective in enhancing stimulus processing. These preferred brain states were considered to be reflexive, with little variation between the four groups suggesting that they were largely determined by the timing of the stimuli.

Barry et al. (2010) shifted paradigms and examined preferred narrow-band EEG phases in an equiprobable auditory Go/NoGo paradigm (sometimes called a 50\% oddball task; Barry et al., 2000) with fixed ISI. This task provides large numbers of stimuli (necessary for phase grouping) in each of two different processing chains: Go 
and NoGo. Note that this paradigm differs importantly from the traditional Go/NoGo task (which has a high proportion of Go trials) and requires minimal inhibition in responding to the NoGo stimulus. Rather, Go responses require effortful processing, while NoGo responses do not (Barry \& Rushby, 2006). All our previous studies in this research stream used digital filtering, but Barry et al. (2010) instead used the Fast Fourier Transformation (FFT) to decompose the ongoing EEG into narrow bands. We again found differential phase occurrence at stimulus onset across the 1-13 Hz narrow EEG bands, and these preferred phase states were linked to more efficient stimulus processing, indicated by latency and amplitude effects in the N1 and P3 components tested. These phase effects differed for Go vs. NoGo stimuli, emphasising their role in the different cortical processing involved. This generalisation of the preferred phase results, to both a new paradigm and a new narrow-band extraction process, considerably strengthened the evidential basis of these effects.

All our previous phase studies sketched above explored the phenomenon in adults. In order to explore the generality of preferred phase states in fixed-ISI paradigms, and their effects on cortical processing, Barry and De Blasio (2012) repeated the Barry et al. (2010) study with a group of children aged 8 to 13 years. Children differ from adults in both their EEG spectrum (Barry \& Clarke, 2009) and ERP morphology in the Go/NoGo task (Johnstone et al., 2005), so this change in age was expected to impact on numerous aspects of the previous results. But as predicted, preferential occurrence of different phase states at stimulus onset was confirmed across our frequency range $(1-13 \mathrm{~Hz})$. In line with developmental expectations, we found that the preferred phase states occurred less in children than in adults, and provided a generally-weaker picture of their effects on ERP components in the Go/NoGo task.

In a parallel series of studies, we began using temporal Principal Components Analysis (PCA) to clarify our understanding of the differential Go/NoGo processing underpinning the components involved in the ERPs obtained in this paradigm. In an adult sample, Barry and De Blasio (2013) identified seven ERP components based on their latency, polarity, and topography: N1, Processing Negativity (PN), P2, N2, P3, the classic Slow Wave (SW), and a diffuse Late Positivity (LP). Based on differential Go/NoGo effects, we proposed that N1 and PN mark the onset of identification of stimulus properties defining Go vs. NoGo, with further sensory processing producing the P2. Identification of the "NoGo" stimulus produces a frontal N2, fronto-central P3a, and enhanced LP; identification of the "Go" stimulus is associated with a parietal N2 and P3b, and SW, together indicating the effortful processing of response preparation and execution. Barry et al. (in press) recently confirmed this processing schema in another adult sample, and extended it to a child group. We use this PCA approach and our Go/NoGo processing schema to framework our understanding of observed phase effects in the ERP components obtained here. 
In the present study we decided to shift our focus, from the narrow 1-Hz bands spanning the 1-13 $\mathrm{Hz}$ range used in our studies described above, to the traditional delta, theta, alpha and beta bands. We have previously examined alpha effects in the fixed-ISI equiprobable Go/NoGo task, which we described then as an auditory oddball task with 50\% target probability (Barry et al., 2004). There was no difference in the occurrence of negativity vs. positivity, but negative driving occurred significantly more often than positive driving, and waxing occurred significantly more often than waning phases. These preferred phases were associated with latency and amplitude effects in N1, N2, and P2, but not P3. For example, N1 latency was reduced in waxing phases, suggesting that this preferred phase state increased processing efficiency.

Unfortunately, there is an additional complication in studies on this aspect of brain dynamics. All previous studies cited above, from the pioneering work of Başar and colleagues 30 years ago up to Barry and De Blasio (2012), possess a common weakness, despite at times herculean efforts to circumvent or reduce its effects. In essence, all these estimate the parameters of a particular frequency at any time from a window that includes data preceding and following that point in time. We do not refer here to the well-recognised "spectral leakage" of data of one frequency into nearby frequencies, commonly encountered in FFT or other frequency separations. Rather, we are concerned by the related, but often unrecognised, leakage of data from one time point into adjacent time points. This smearing of data, particularly between the prestimulus and poststimulus period, is inherent in all forms of time-frequency decomposition, whether analog or digital filtering, or wavelet or FFT spectral separations. This means that estimates of EEG activity around the time of stimulus onset are necessarily contaminated by contributions from the immediately-poststimulus EEG, affecting parameter estimates to an unknown extent. For example, estimates of the phase/amplitude of EEG bands at stimulus onset are commonly derived from FFT or wavelet analysis of a data window centred at time zero, and include EEG elements from the prestimulus data for half the window length, and from the poststimulus data for the other half window length. This poststimulus half window includes stimulus-induced activity, whether this is evoked activity or re-aligned ongoing EEG contributing to the ERP (Barry et al., 2009). The major poststimulus ERP components (N1/P3) are of fixed polarity and relatively fixed in time, and even their partial inclusion in prestimulus FFT estimates will contribute systematically to artefactual estimates of phase at stimulus onset. We uniquely avoided this problem here by calculating EEG phase before the stimulus onset, using a data window ending at stimulus onset. We then extrapolated this phase forward to the time of stimulus onset, and based our phase separations on this extrapolated phase value.

Because no previous phase study of EEG bands (whether narrow 1-Hz bands or traditional alpha etc. bands) has avoided the bleeding of poststimulus EEG into the prestimulus period, thus confounding the valid estimation of prestimulus phase, there is no existing artefact-free data to generate hypotheses for this study. 
Essentially, the aim of this study is to provide, for the first time, valid artefact-free data on the existence of preferred phase states at stimulus onset, and their effects on poststimulus brain functioning as reflected in phaseselected ERP components. If the artefacts we avoid are large, the new data will be quite dissimilar to the existing literature; if small, we will refine previous findings. We have no way to estimate the extent of the artefacts $a$ priori, but will probe this in the study.

\section{Materials and methods}

\subsection{Participants}

Twenty-two university students initially participated in this study as one means of satisfying a course requirement, but 2 were later excluded on the basis of poor performance (see section 2.3). The remaining 20 participants (15 females and 5 males) were aged between 18 and $36(M=21.6, S D=3.8$ years); 18 were right handed. All were screened for neurological diseases, head injuries, and psychiatric conditions, claimed normal hearing and normal or corrected vision, and were required to abstain from caffeine and other psychoactive substances for at least $4 \mathrm{~h}$ prior to the study. Participation was voluntary and written informed consent was obtained following a protocol approved by the joint University of Wollongong/South East Sydney and Illawarra Area Health Service Human Research Ethics Committee, in accordance with the Declaration of Helsinki.

\subsection{Procedure}

EEG was recorded from 19 scalp sites using an electrode cap with tin electrodes, referenced to the left ear, and the right ear channel was recorded separately. Vertical electro-oculogram (EOG) was recorded from electrodes above and below the left eye, and horizontal EOG from electrodes beyond the outer canthi. All impedances were below $5 \mathrm{k} \Omega$. Data were continuously sampled with a Neuroscan Synamps 2 digital signal-processing system with Neuroscan Acquire software (Compumedics, Version 4.3.1) at a rate of $1000 \mathrm{~Hz}$, with a gain of 500, and recorded using a $30 \mathrm{~Hz}$ lowpass filter for off-line analysis.

Subjects were seated in a comfortable chair within an air-conditioned sound-reduced room located adjacent to the room housing the recording equipment. Four blocks of an auditory Go/NoGo task were presented to each subject via circumaural headphones. Each presentation block consisted of 150 tones of $60 \mathrm{~dB}$ SPL, $50 \mathrm{~ms}$ duration and $5 \mathrm{~ms}$ rise/fall times, presented with a fixed SOA of $1100 \mathrm{~ms}$. Half the tones were $1000 \mathrm{~Hz}$ (tone A) and half were $1500 \mathrm{~Hz}$ (tone B), with these delivered in a randomised order. The participants were required to button-press to one of the tones; the target frequency alternated between the blocks (i.e., ABAB or BABA), and the 
frequency of the target tone of the first block was counterbalanced across participants. Participants were asked to remain fixated on a small cross displayed on a computer monitor (LCD) $1 \mathrm{~m}$ in front of them, and also to refrain from blinking. Each completed a brief EOG calibration task prior to the experiment.

\subsection{EEG processing}

Figure 2 shows a schematic outline of the major stages of EEG processing, emphasising the novel phaserelated aspects. First, the RAAA EOG Correction Program (Croft \& Barry, 2000) was applied to correct the EEG data, after which the continuous data were re-referenced to digitally-linked ears, and low-pass filtered (FIR: 0.1-25 Hz, zero-phase shift, $24 \mathrm{~dB} /$ Octave). Single-trial epochs ( -1100 to $+1100 \mathrm{~ms})$ were extracted for each stimulus. Those trials containing muscular or other artefact, or incorrect responses (commission errors to NoGo trials, and omissions or RTs > 500 ms to Go trials) were identified and excluded from further analysis. The datasets of two participants were discarded due to substantial differences in their error rates to Go compared to NoGo (18.3 vs. 1.7 $\%$ and 2.3 vs. $16.3 \%$ ). The remaining participants had Go/NoGo error rates below $9 \%$ (Go: $M=3.1, S D=2.1 \%$; NoGo: $M=2.4, S D=2.1 \%)$.

Fig. 2 about here

For each accepted (i.e., artefact- and error-free) trial of each accepted subject, EEG post-processing was carried out separately within MATLAB ${ }^{\circledR}$ (R2012b, The MathWorks, Natic, MA). For each trial and site, the CNV amplitude was defined as the difference between mean amplitudes in the (-500 to $-250 \mathrm{~ms})$ and (-100 to $0 \mathrm{~ms})$ prestimulus ranges. Pre- and poststimulus RMS amplitudes were calculated from the band-selected inverse FFTs derived from FFTs of unpadded 10\% Hanning-windowed 500 ms wide-band epochs.

In general, for phase determinations, we converted EEG data at $\mathrm{Cz}$ from the time domain to the timefrequency domain by applying an FFT to a 10\% Hanning window over a 250 ms epoch, with zero padding to extend the window size to $500 \mathrm{~ms}$; the frequency resolution of the FFT was thus $2 \mathrm{~Hz}$. For each frequency bin from $\mathrm{n}=2$ to $24 \mathrm{~Hz}$, the magnitude and phase were calculated (after scaling to compensate for the Hanning window) at the centre of that window. For the phase of each frequency n, this required the value at the beginning of the window (as calculated by the FFT) to be adjusted to the centre of the window by shifting its phase forward $125 \mathrm{~ms}$ (i.e., $\mathrm{n} \times 125 / 1000$ cycles) by adding $(2 \pi \times \mathrm{n} \times 125 / 1000=\mathrm{n} \pi / 4)$ radians. Note that all phase data reported here use the circular notation shown on the right in Figure 1, and have been transformed to match our previous convention defined on the left of this figure.

To illustrate our processing approach, Figure 3 shows data from a randomly-selected Go trial (32) of a randomly-selected subject (S08) for the two frequencies contributing to theta, $\mathrm{n}=4$ and $6 \mathrm{~Hz}$ (columns 1 and 2 
respectively). We plot FFT magnitude, FFT phase and the inverse-FFT amplitude, where amplitude $=$ magnitude $\times$ cos(phase). The first FFT window was centred at $-500 \mathrm{~ms}$ (i.e., utilising data from -625 to -375 ms), and generated the first data point in each of the top 3 plots for the first two columns. This procedure was repeated, stepping forward in time by $1 \mathrm{~ms}$, until the decomposition reached a central point of $+500 \mathrm{~ms}$; i.e., this trial for illustration required 1001 separate FFTs. In the time-frequency plots of the first three rows, the central vertical black line marks time 0 , stimulus onset. The first row shows the magnitude at each frequency as a function of time, and some increase in both 4 and $6 \mathrm{~Hz}$ activity is apparent after stimulus onset. The dominance of the ongoing regular phase increasing with time is apparent in the second row as a diagonal increase, interrupted by the wrap-around of phase at $2 \pi$ (vertical drop to 0 radians) occurring approximately $n$ times during the 1 -s epoch. It can be seen that this regularity is disrupted by phase resets, one of the mechanisms involved in ERP genesis (Barry, 2009). Four of the more-obvious phase resets are marked by red circles on the left ( $4 \mathrm{~Hz}$ ) panel of the second row, two in the prestimulus period and two in the poststimulus period.

Fig. 3 about here

For each accepted trial at $\mathrm{Cz}$ in each condition, for each $2 \mathrm{~Hz}$ bin at frequency $\mathrm{n}$, the phase at stimulus onset was estimated by extrapolation from the last prestimulus data point unarguably uncontaminated by poststimulus activity - i.e., the phase of the epoch centred at $-125 \mathrm{~ms}$, marked by a dashed vertical line in the top three rows of Figure 3. This extrapolation required a further addition of $n \pi / 4$ radians, as illustrated in the bottom row of Figure 3. Here the dashed line to the unfilled mark on the unit circle indicates the observed phase at -125 ms, and the full black line to the black mark indicates the estimated phase at stimulus onset. For $n=4 \mathrm{~Hz}$, this is $\pi$ radians later; for $\mathrm{n}=6 \mathrm{~Hz}$, it is $3 \pi / 2$ radians later.

The magnitudes and phases of the individual frequency bins were combined into the magnitude and phase for each of the four bands (delta: $2 \mathrm{~Hz}$; theta: 4-6 Hz; alpha: 8-12 Hz; beta: 14-24 Hz) using vector addition to take account of the instantaneous magnitude and phase angle of each contributing narrow band. This uses the Pythagorean theorem to compute the magnitude, and the arctan to compute the phase, of pairs of frequencies being combined (see e.g., http://www.1728.org/vectutor.htm). The plots in the third column of Figure 3 illustrate the result of this process (for this trial) for the theta band. The estimated phase at stimulus onset was then used to categorise the epoch into one of four groups (labelled A, B, C and D), corresponding to the four phase ranges shown in Figure 1: $0-\pi / 2, \pi / 2-\pi, \pi-3 \pi / 2$, and $3 \pi / 2-2 \pi$. For the individual Go trial shown here, the estimated theta phase at stimulus onset falls within phase quadrant A. This procedure was followed to obtain phase information at stimulus onset for each of our four bands for each trial of each subject. Unlike the large number of FFTs required to generate our illustrations of individual data (e.g., Figure 3), this required only two FFTs per trial per subject: 
centred at -125 ms as input to our computation of the estimated phase at stimulus onset, and centred at 0 ms to compute the observed phase at stimulus onset.

For each band we also compared the estimated phase at stimulus onset with the observed phase at stimulus onset in order to assess the extent of the artefact associated with the uncorrected phase. For the trial shown in Figure 3, the FFT phase plot indicates that the observed theta phase at stimulus onset is also within the range for classification as phase A (i.e., $0-\pi / 2$ ); however, the value of the observed phase angle is notably less than the estimated phase value seen in the bottom row. We calculated the circular correlation between the estimated and observed phase pairs from each accepted trial for each subject, and examined this across subjects.

Subsequent phase-related processing was based on the estimated $\mathrm{Cz}$ phase angles at stimulus onset. The proportion of trials falling into each phase group was recorded for later analysis, and the pre- and post-stimulus root mean square (RMS) band amplitudes, and CNV amplitudes, were computed at nine core sites (F3, Fz, F4, C3, Cz, C4, P3, Pz, P4). ERPs were derived from the wide-band trials after the phase group sub-division was applied to all 19 sites; this was done separately for each of the defined bands. For each subject for each of the delta, theta, alpha and beta bands, this produced four averaged wide-band (0-25 Hz) ERPs at each site, differing in the estimated phase (A, B, C, D) at stimulus onset at Cz, for each stimulus type (Go, NoGo). All these ERP epochs commenced $100 \mathrm{~ms}$ prestimulus, included $850 \mathrm{~ms}$ of data, and were baselined across the $100 \mathrm{~ms}$ period immediately prestimulus.

These 16 sets of average ERPs ( 4 bands $\times 4$ phase divisions) and the grand mean Go/NoGo datasets were exported to the ERP PCA toolkit (version 2.23; Dien, 2010) in MATLAB. For each PCA, epochs -100 - 750 ms, containing 850 data points, were used. To define topography and Go/NoGo effects, we first conducted an overall PCA on the grand mean data across all accepted trials, independent of band and phase. For this PCA we had 19 sites $\times 20$ subjects $\times 2$ conditions, or 760 cases, so we quarter-sampled the data in the epoch to 212 points (variables), for a case:variable ratio 3.6. To investigate phase effects, we conducted four separate PCAs, one for each band, each containing Go and NoGo ERPs at the four phases. With 19 sites $\times 20$ subjects $\times 4$ phases $\times 2$ conditions, each band PCA had 3040 cases; we half-sampled these data to 425 points to reduce computation time, yielding a case/variable ratio 7.2. Each temporal PCA was based on the covariance matrix, and used an unrestricted Varimax rotation (i.e., all factors were rotated) with Kaiser normalisation, following Kayser and Tenke (2003). Dien's (2010) toolkit conveniently provides the component amplitude waveforms for each subject, condition and electrode site. These are the product of the factor loadings, scaled to microvolts by multiplication with the standard deviation in the raw dataset for each time-point, and the factor score for the particular subject, condition, and electrode site. Factor identification involved consideration of the component latency, polarity, and 
peak amplitude topography in the context of our previous PCA work in this paradigm (Barry \& De Blasio, 2013; Barry et al., in press).

We also used the coefficient of congruence (Tucker, 1951) to compare components in the four band PCAs to the corresponding components in the grand mean PCA. This is a PCA-relevant variant of correlation of the unscaled factor loadings over time, without the usual mean correction. It compares the temporal waveform of components, and is sensitive to differences in peak latency, onset/offset times, and relative magnitudes, but not topography. The similarity of two components is assessed by a rule-of-thumb: $r_{c}<.85$ indicates no factor similarity, .85 $<r_{c}<.94$ indicates fair similarity, and $r_{c}>.95$ indicates equality (Lorenzo-Seva \& ten Berge, 2006).

\subsection{Statistical analyses}

CNV amplitudes, prestimulus RMS amplitude for each band (delta, theta, alpha, and beta), and RMS band changes associated with stimulus onset, were examined in separate two-way MANOVAs, assessing amplitudes at nine core sites (F3, Fz, F4, C3, Cz, C4, P3, Pz, P4) with the within-subjects factors of Topography and stimulus Type (Go vs. NoGo). Within Topography, sagittal plane (frontal [F3, Fz, F4], central [C3, Cz, C4] and parietal [P3, Pz, P4] regions) and coronal plane (left [F3, C3, P3], midline [Fz, Cz, Pz] and right [F4, C4, P4] regions) were repeated-measures factors. Planned contrasts within the sagittal plane compared frontal vs. parietal regions, and central sites vs. the mean of the frontal and parietal sites. Within the coronal plane, the left vs. right regions, and the midline vs. the mean of the left and right sites, were analysed. These orthogonal planned contrasts and their interactions provide optimal information on the topographic distribution of each measure. Components from the PCA on the grand mean data across all trials were analysed similarly to provide general across-band information.

To assess the occurrence of preferred brain states, circular statistics were examined using the CircStat MATLAB toolbox (Berens, 2009). We report results of the Hodges-Ajne Omnibus test for non-uniformity of circular data. This tests for non-uniformity of circular distributions and makes no assumption about the nature of departures from uniformity, being appropriate for unimodal, bimodal and multimodal data. Separately for each band and stimulus condition, this assessed the phase distribution across the accepted trials for all subjects. In addition, the within-subjects proportion of trials identified in each phase range was obtained for each band and analysed with a two-way repeated-measures MANOVA. Three orthogonal Phase contrasts were examined, representing the conceptualisation described earlier (negativity vs. positivity, negative driving vs. positive driving, waxing vs. waning), as was a contrast examining the effect of stimulus Type (Go vs. NoGo), and their interaction.

Phase effects in Go reaction time (RT) were examined in a one-way ANOVA over Phase, using the above orthogonal phase contrasts. The amplitudes of the identified PCA components were analysed independently for Go 
and NoGo responses in separate two-way repeated-measures MANOVAs, as a function of Topography and Phase, using the Topography and Phase contrasts outlined above.

The problems of multiple testing were carefully considered given the extensive statistical analyses involved in this study. For each of the four frequency bands, the contrasts in each analysis of a particular measure were planned, and there were fewer of them than the degrees of freedom for effect; hence Bonferroni-type adjustments to $\alpha$ were unnecessary (Tabachnick \& Fidell, 1989). However, as the effects in a particular measure were assessed via repeated testing at 4 bands, this increase in Type I error probability was controlled. Instead of applying a Bonferroni correction, which is quite conservative, we adopted the false discovery rate (FDR) control procedure of Benjamini and Hochberg (1995) for independent tests. Commencing with the desired $\alpha$ (here .05), the FDR control procedure linearly decreases the significance threshold to $\alpha / \mathrm{m}$, where $\mathrm{m}$ is the number of tests (here 4 , yielding: .05, .0375, .025, .0125). Observed $p$ values (for the 4 levels of band) are placed in descending order and sequentially compared. The first (initially) significant effect to satisfy the adjusted threshold, and all those following, remain significant. We used the FDR function based on Benjamini and Yekutieli (2001) provided in EEGLAB (rev. 9.0.8.6b; Delorme \& Makeig, 2004). Here we applied this procedure across the 4 bands for each corresponding effect or interaction.

We also assess phase effects in a number of ERP components, each of which may be considered to constitute a separate experiment. The increased number of 'experiments' increases the frequency, but not the probability, of Type I errors. For example, within 20 significant results for any one variable, the corrected Type I error rate of .05 means that 1 is likely to be a false positive. This likelihood of 1 error in each 20 significant tests remains if we assess two variables (e.g., N1-1 and PN amplitude) - although considering the second set of (say) 20 significant tests increases the expected frequency of Type I errors to 2, the probability is unchanged (2 in 40 tests = .05). That is, the probability of Type I error is not increased, and Howell (1997) argues that the increase in error frequency cannot be “controlled” by adjusting alpha levels. This is an important distinction - here we use FDR to correct for multiple testing within a measure, but not for testing a number of different measures.

All $F$ tests reported here had $(1,19)$ degrees of freedom. The problems of non-sphericity often encountered with repeated-measures analyses of physiological variables are precluded by our single degree of freedom contrasts, and hence there is no need for their control using Greenhouse-Geisser type epsilon adjustments (O’Brien \& Kaiser, 1985). In order to save space, only phase-related effects are reported at length, as these were of primary interest. We also report effects that approach significance regarding phase occurrence (only) to encourage further exploration, but do not discuss them. 


\section{Results}

\subsection{RMS amplitudes of EEG activity in the pre- and post-stimulus epochs}

Prestimulus RMS amplitudes at the analysed sites are shown for Go and NoGo separately as a function of the $2 \mathrm{~Hz}$ narrow-band frequencies in panel A of Figure 4, with the band ranges indicated at Pz. Across stimuli, delta was topographically dominant in the midline $(p<.001)$, theta was topographically dominant in the midline $(p$ $<.001$ ), particularly centrally (i.e., in the vertex, $p=.003$ ); alpha was topographically dominant in the midline ( $p<$ $.001)$ and strongly parietal (F $<\mathrm{P}: p<.001 ; \mathrm{C}<\mathrm{F} / \mathrm{P}: p=.006)$; and beta was also midline $(p=.015)$. There was no main effect of Go vs. NoGo in delta, theta, alpha, or beta; the prestimulus spectra in Figure 4 overlap at all sites, and there were no differences in prestimulus topography between the conditions.

Fig. 4 about here

Poststimulus increases in RMS amplitudes are shown as a function of the $2 \mathrm{~Hz}$ narrow-band frequencies in Figure 4 panel B for the analysed sites. Increases in delta were larger centrally $(p<.001)$, particularly in the right hemisphere $(p=.007)$; theta increases were larger frontally $(p<.001)$ and centrally $(p<.001)$, and larger in the midline $(p<.001)$, particularly frontally $(p=.001)$ and centrally (i.e., in the vertex, $p=.001)$; alpha increases were dominant frontally $(p=.003)$; and centrally $(p<.001)$, particularly in the right hemisphere $(p=.018)$, and in the midline $(p=.012)$; but beta showed a decrease that was larger frontally $(p=.014)$ and in the left central region $(p=$ .019). There were Go vs. NoGo effects on these changes in RMS amplitudes that differed between the bands. In delta, increases in Go were greater in parietal regions $(p<.001)$, particularly the midline $(p<.001)$, and in the right hemisphere $(p=.006)$, particularly centrally $(p=.003)$, with a smaller increase at the vertex $(p=.011)$. There were no Go/NoGo differences in theta. In alpha, there was an overall decrease in Go and increase in NoGo ( $p=$ $.004)$; the decrease in Go was larger parietally $(p=.016)$ and in the left hemisphere $(p=.022)$. The decrease in beta was larger for Go than NoGo $(p<.001)$; particularly in the left hemisphere $(p=.007)$ and frontal midline $(p=$ $.002)$.

\subsection{Grand mean ERPs}

Across the 20 subjects, the number of accepted epochs ranged from 268 to $294(M=283.1, S D=8.36)$ for the Go stimuli, and from 255 to $298(M=285.3, S D=10.8)$ for the NoGo stimuli. Figure 5 (left) illustrates the mean Go and NoGo ERPs at the midline sites. Poststimulus, a P1 is visible at around $50 \mathrm{~ms}$, with a frontocentral N1 at approx. 100 ms, and a large P3 between 250 and 400 ms. The typical P3 distribution in this task, a parietally distributed Go P3b and the anteriorisation of the NoGo P3a (Barry \& Rushby, 2006), is clearly evident. P2 ( 200 
ms) and N2 ( 230 ms) peaks are also apparent between N1 and P3. The P3 is followed by a classic frontalnegative/parietal-positive slow wave (SW) peaking around 450 ms, and a Late Positivity (LP) dominant in NoGo around $600 \mathrm{~ms}$.

Fig. 5 about here

As indicated by the statistics in Table 1, the prestimulus CNV was larger centrally and in the right hemisphere; greater parietally in the midline, and centrally in the right hemisphere. It did not differ with Go/NoGo.

\subsection{Grand mean and band PCA outcomes}

Of the 212 temporal factors extracted from the grand mean ERPs, the first 8 explained $93 \%$ of the variance. The sums of these components at the midline sites are displayed in the right panels of Figure 5; comparison with the raw ERPs (left panels of Figure 5) confirms good fit with the original data. In the top panel of Figure 6, the topographic headmaps of the temporal components are displayed averaged across condition, and presented above the loadings of the factors plotted against time. These loadings represent the correlations between the component and the ERP waveform (Tabachnick \& Fidell, 1989), scaled by the standard deviation of each data point in the ERP to obtain loadings in microvolts. The percentage of the total variance accounted for by each rotated component is also indicated. The dominant components (factors 1-8, contributing to the virtual ERPs) were tentatively identified in terms of sequence and latency and our previous work in this paradigm (Barry \& De Blasio, 2013; Barry et al., in press) as P1, N1-1, Processing Negativity (PN), P2, N2, P3, the classic SW, and LP. The separate Go and NoGo topographic headmaps of the assessed components are displayed in the bottom panel of Figure 6.

Fig. 6 about here

The same eight components identified in the grand mean PCA were found in each band PCA, and these explained 72 to $85 \%$ of the variance of each set. Apart from low $r_{c}$ for P1 and N2, all components showed fair similarity $\left(r_{c}>.85\right)$ or equivalence $\left(r_{c}>.95\right)$ to the grand mean components shown in Figure 6 . The factor loadings for P1 and N2 are also rather small, and somewhat "hidden” in the other loadings (see Figure 6), so we decided to exclude them from further consideration here.

As shown in the headmaps of Figure 6, the grand mean statistics listed in Table 1 indicate that N1-1 was frontocentral with a midline and right-frontal dominance; and was enhanced for Go compared with NoGo. PN was larger in the hemispheres than the midline. This is the first example of the underlining used to indicate an effect

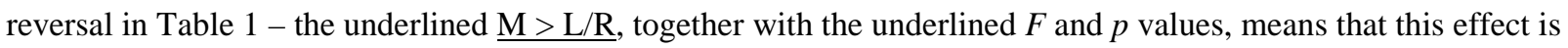
reversed (i.e., $\mathrm{M}<\mathrm{L} / \mathrm{R}$ ) for PN. PN was larger on the right, and smallest at the vertex; overall, $\mathrm{PN}$ was more 
negative to NoGo than Go, with frontocentral and midline enhancements; these interacted, with the frontocentral enhancements being larger in the midline. P2 was more positive centrally and in the left hemisphere, particularly centrally; overall, P2 was enhanced to Go, and particularly so in the midline, and in the midline frontal and vertex regions. P3 was centroparietal with a midline dominance; these effects interacted, with the centroparietal enhancements larger in the midline; the central enhancement was dominant on the right. P3 to Go was parietal, less midline- and more right-hemisphere-dominant (particularly centrally), while P3 to NoGo was central and midline (particularly frontally), with a vertex maximum, contributing to an overall larger NoGo P3. SW was frontalnegative/parietal-positive with central and hemispheric enhancements; the frontal/parietal difference was enhanced in the midline, and the central enhancement was larger in the hemispheres. Go was associated with greater positivity in SW, particularly centroparietal and midline enhancements, the latter particularly in the parietal region, and a left parietal increase. LP was greater centrally and in the right hemisphere; these interacted, leading to a right central dominance with frontal enhancements in the hemispheres. NoGo LP was enhanced in the posterior and in the left hemisphere, in the left-posterior, left-central, and hemispheric-central regions, resulting in an overall more positive LP to NoGo, Phase effects in these components were examined in the separate band PCAs and are described later.

Table 1 about here

\subsection{Preferential occurrence of EEG phases at stimulus onset}

The preferential occurrence of the different phases at stimulus onset is illustrated in the circular phase histograms of Figure 7 for the Go data of our illustrative participant (S08). The top row shows the observed phase distribution over all accepted trials obtained for each band at $-125 \mathrm{~ms}$, and the middle row shows the distribution of the actual/observed phases at stimulus onset (corresponding to previous phase studies). The bottom row shows the uncontaminated estimated/predicted phases at stimulus onset. For delta in this subject, the circular median phase angle was 0.72 rad (phase A); this dominant occurrence of phase A is clearly apparent (159/268 accepted trials), and it can be seen that the overall distribution differed significantly from uniformity/randomness $(p<.001)$. In theta, the circular median phase angle was 2.68 rad (phase B); the dominant occurrence of phase B (98/268) is evident, and again the distribution departed from uniformity $(p=.007)$. In alpha, the circular median phase angle was 2.60 rad (phase B); the dominant occurrence of phase B (84/268) is apparent, and overall the distribution was non-uniform ( $p=.011)$. In beta, the circular median phase angle was 3.80 rad (phase C); phase C was clearly dominant (98/268), and the distribution again differed significantly from randomness $(p=.023)$.

Fig. 7 about here 
In Figure 8, the amplitudes for each successful Go trial from this subject are displayed as a function of time in the top section of panel a, with the resultant mean ERP across the trials shown below. Panel a presents the wide-band (0-24 Hz) EEG for all accepted Go trials, in order of stimulus presentation. Panel b presents the corresponding alpha data stream, with the trials ordered according to their estimated phase at stimulus onset, while panel d shows the wide-band EEG trial data (as in panel a) sorted in terms of the alpha phase at stimulus onset (i.e., trials ordered as in panel b). The trial divisions for phases A, B, C, and D at stimulus onset are also indicated in panels $b$ and $d$. The non-random distribution of the trials across the four phases is apparent and, in this participant, the preferential occurrence of phases B and C (together: positive driving) in alpha is again clearly evident (c.f.

Figure 7). The mean ERPs derived from the alpha data for the phase sorted trials in each of the four phase ranges are displayed in panel c (compare phase at time 0 with Figure 1 (left) phase definitions). The mean ERPs derived from the corresponding raw EEG data for each phase are shown in panel e, where (for example) the N1 activity is noticeably predominant in phases A and B (negativity).

Fig. 8 about here

The omnibus test across all subjects indicated the lack of circular uniformity in both Go and NoGo trials for delta and theta (all $p<.001)$, and gave some support for non-uniformity for Go trials in alpha $(p=.057)$. In terms of our more-sensitive preferred phase groupings, across all subjects, negativity occurred significantly more often than positivity in the delta band $(p<.001)$. This and all other negativity vs. positivity effects are shown in Table 2 by directional arrows indicating an increase/decrease, and asterisks indicating the level of significance. Negative driving occurred significantly more often than positive driving phases in delta $(p=.001)$, and less often in theta $(p=.001)$ and alpha $(p=.003)$, and somewhat less often in beta $(p=.059)$; see Table 3 for these and all other negative driving vs. positive driving effects. Waxing occurred significantly more often than waning phases in the delta $(p<.001)$ and beta $(p<.001)$ bands, and less often in theta $(p<.001)$ and alpha $(p<.001)$; see Table 4 for all waxing vs. waning effects. The occurrence of these preferred phases (main effects as listed above) was substantial, with the eight significantly-preferred phases occurring some 8-388 \% more/less often than the non-preferred phases, with an average of some $67 \%$. These preferred phases did not differ between Go and NoGo, except in beta, where negativity occurred somewhat more often in NoGo than Go $(p=.080)$, and waxing occurred more often in Go than NoGo $(p=.010)$; note that this last difference was small - some $4 \%$ of trials.

Tables 2, 3, 4 about here

\subsection{Phase effects}

\subsubsection{Stimulus onset phase and RT}


Although RT was shorter for Negativity phases (360.8 ms) compared with positivity phases (369.8 ms) in delta (at $p=.014$ ), the significance of this effect did not survive the FDR procedure. There were no other phase effects on RT.

\subsubsection{Relation between stimulus onset phase and RMS amplitudes}

Negativity phases were associated with larger prestimulus RMS amplitudes in delta in the right hemisphere $(p<.001)$ (indicated in Table 2 by the upward arrow in the delta column next to "phase $\times \mathrm{L}<\mathrm{R}$ " [read as "phase increases R more than L"]), particularly centrally $(p=.004)$ (indicated by the upward arrow next to "phase $\times \mathrm{C}>$ $\mathrm{F} / \mathrm{P} \times \mathrm{L}<\mathrm{R}$ " [read as "phase increases $\mathrm{R}$ more than $\mathrm{L}$ in central c.f. F/P regions]"). Negative driving phases were associated with decreased prestimulus RMS amplitudes in delta centrally $(p=.002)$; and in theta centrally $(p=$ $.001)$, particularly in the right hemisphere $(p=.001)$. Waxing vs. waning phases were associated with larger prestimulus RMS amplitudes in delta $(p<.001)$, particularly centrally $(p=.001)$, in the midline $(p<.001)$, and at the vertex $(p=.007)$; in alpha $(p=.016)$, particularly in the midline $(p=.001)$; and in beta in the midline $(p=$ .035); and with smaller amplitudes in theta $(p=.028)$, particularly centrally $(p=.002)$, in the midline $(p=.002)$, and at the vertex $(p=.006)$.

There was a general poststimulus increase in RMS amplitude in delta, theta, and alpha, but a decrease in beta. There were no effects of negativity phases on RMS amplitudes in any band. Negative driving phases increased RMS amplitudes centrally in delta $(p=.017)$; and in theta $(p=.021)$, particularly in the right hemisphere $(p=.001)$. Waxing vs. waning phases were associated with smaller increases in poststimulus RMS amplitudes in delta in the midline $(p=.018)$; with larger increases in theta centrally $(p=.021)$ and in the midline $(p=.002)$; and with larger poststimulus decreases in RMS amplitude in beta in the midline ( $p=.034$ ), and in the left hemisphere in Go $(p=.007)$.

\subsubsection{Relation between stimulus onset phase and CNV amplitude}

Negativity phases in delta were associated with larger CNV amplitudes $(p<.001)$, particularly centrally $(p$ $<.001)$ and in the midline $(p<.001)$; but with smaller CNVs in theta $(p<.001)$, more so centrally $(p<.001)$ and in the midline $(p=.005)$; in alpha $(p=.003)$, particularly in the midline $(p=.022)$; and in beta $(p<.001)$, particularly centrally $(p=.009)$ and in the midline $(p=.005)$. Negative driving phases were associated with increased CNVs in delta $(p<.001)$, particularly centrally $(p<.001)$ and in the midline $(p<.001)$; and in beta $(p<$ $.001)$, particularly centrally $(p<.001)$ and in the midline $(p=.001)$; but with smaller CNVs in theta $(p<.001)$, more so centrally $(p<.001)$ and in the midline $(p<.001)$; and in alpha $(p<.001)$, particularly centroparietally $(p$ 
$=.003$ and $p=.005$, respectively), and in the midline $(p<.001)$. The negative driving changes were largest at the vertex in delta $(p=.005)$ and theta $(p=.006)$. There were no main effects of waxing vs. waning phases on CNV amplitudes.

\subsubsection{Phase at stimulus onset effects on ERP components}

\subsubsection{N1-1}

Go N1-1 amplitudes in negativity compared with positivity phases were significantly larger in theta ( $p=$ $.011)$, particularly frontally $(p=.035)$, in alpha parietally $(p=.006)$, and in beta frontally $(p=.020)$; and significantly smaller in delta $(p=.002)$. Amplitudes for Go N1-1 in negative driving compared with positive driving phases were significantly larger in theta $(p<.001)$, particularly in the midline $(p<.001)$, and parietal and central midline ( $p=.012$ and $p=.004$ respectively); and reduced in beta $(p=.023)$, particularly parietally $(p=$ .004). Waxing vs. waning phases were not associated with Go N1-1 effects.

NoGo N1-1 amplitudes in negativity compared with positivity phases were significantly smaller in delta ( $p$ $=.004)$; and larger in theta $(p=.024)$, particularly frontally $(p=.010)$. Amplitudes for NoGo N1-1 in negative driving compared with positive driving phases were significantly larger in theta $(p<.001)$, particularly in the midline $(p<.001)$ and vertex $(p=.012)$. Waxing vs. waning phases were not associated with differences in NoGo N1-1 amplitudes.

\subsubsection{2. $P N$}

The only phase effect on Go PN amplitudes that survived the FDR procedure was in negativity compared with positivity phases, where Go PNs were significantly smaller in beta frontally $(p=.002)$.

NoGo PN amplitudes in negativity compared with positivity phases were significantly smaller in delta $(p=$ .005); and frontally smaller in beta $(p=.007)$. Amplitudes for NoGo PN in negative driving compared with positive driving phases were significantly larger at the vertex in alpha $(p=.010)$. Waxing vs. waning phases were associated with smaller NoGo PN amplitudes in the central right region in delta $(p=.005)$; and in the parietal midline in alpha $(p=.010)$.

\subsubsection{3. $P 2$}

Go P2 amplitudes were not affected by negativity compared with positivity phases. Amplitudes for Go P2 in negative driving compared with positive driving phases were significantly larger frontally in theta $(p=.010)$ and on the right in alpha $(p=.008)$. Waxing vs. waning phases were associated with larger Go P2 amplitudes frontally 
in delta $(p=.010)$; and with larger amplitudes in the left frontal region in theta $(p=.004)$.

The only phase effect in NoGo P2 amplitudes to survive the FDR procedure was that negative driving compared with positive driving phases were associated in delta with enhanced NoGo P2 amplitudes in the right central region $(p=.005)$.

\subsubsection{P3}

Go P3 amplitudes in negativity compared with positivity phases were significantly enhanced in delta $(p<$ $.001)$, particularly centrally $(p=.002)$ and in the midline $(p<.001)$; and significantly reduced in theta $(p=.008)$, particularly centrally $(p=.014)$. Amplitudes for Go P3 in negative driving compared with positive driving phases were significantly larger in beta $(p=.002)$; but smaller in theta $(p<.001)$, particularly centrally $(p=.002)$, in the midline $(p<.001)$, and at the vertex $(p=.002)$. Waxing vs. waning phases were not associated with Go P3 effects that survived the FDR procedure.

NoGo P3 amplitudes in negativity compared with positivity phases were significantly larger in delta $(p<$ $.001)$, particularly centrally $(p=.003)$, in the midline $(p<.001)$, and at the vertex $(p=.005)$; but were reduced in beta in the posterior hemispheres $(p=.006)$. Amplitudes for NoGo P3 in negative driving compared with positive driving phases were significantly enhanced in delta $(p=.001)$, particularly centrally $(p=.005)$, and in the midline $(p=.002)$; and in beta $(p=.005)$; but reduced in theta $(p<.001)$, particularly centrally $(p<.001)$, in the midline $(p$ $<.001)$, and at the vertex $(p=.006)$. Waxing vs. waning phases were associated with enhanced NoGo P3 amplitudes in delta in the left central region $(p=.012)$.

\subsubsection{SW}

The Go SW amplitudes in negativity compared with positivity phases were significantly more positive in delta in the midline $(p=.010)$; and significantly less positive in beta at the vertex $(p=.012)$. Amplitudes for Go SW in negative driving compared with positive driving phases were significantly more positive in delta $(p<.001)$, particularly on the right $(p=.002)$; but more negative in theta $(p<.001)$, particularly centrally $(p<.001)$, and in the midline $(p<.001)$. Waxing vs. waning phases were not associated with Go SW effects.

The overall NoGo SW amplitudes in negativity compared with positivity phases were significantly more positive in delta $(p<.001)$, particularly in the midline $(p=.006)$ and the central right region $(p=.003)$. Amplitudes for NoGo SW in negative driving compared with positive driving phases were significantly more negative in theta $(p<.001)$, particularly in the central $(p=.010)$ and midline $(p=.001)$ regions; and less negative in beta $(p=.006)$. Waxing vs. waning phases were not associated with effects in NoGo SW amplitudes. 
The Go LP amplitudes in negativity compared with positivity phases were significantly more positive in delta $(p<.001)$, particularly centrally $(p=.003)$ and in the midline $(p<.001)$; and significantly more negative in theta ( $p=.019)$, and in alpha $(p=.017)$. Amplitudes for Go LP in negative driving compared with positive driving phases were significantly more positive in delta $(p=.003)$, particularly centrally $(p=.007)$; and in beta $(p=.002)$; but more negative in theta $(p<.001)$, particularly centrally $(p<.001)$, and in the midline $(p<.001)$. Waxing vs. waning phases were not associated with Go LP effects.

The overall NoGo LP amplitudes in negativity compared with positivity phases were significantly more positive in delta $(p<.001)$, particularly centrally $(p=.001)$ and in the midline $(p=.001)$; but less positive in theta centrally $(p=.025)$. Amplitudes for NoGo LP in negative driving compared with positive driving phases were significantly more positive in delta $(p<.001)$, particularly in the central $(p=.002)$ and midline $(p=.001)$ regions; but more negative in theta $(p<.001)$, particularly in the central $(p<.001)$ and midline $(p<.001)$ regions. Waxing vs. waning phases were not associated with effects in NoGo LP amplitudes.

\subsection{Extent of artefact in uncorrected phases}

For our illustrative subject, Figure 7 shows the difference between the observed uncorrected phase estimates previously reported in the literature (middle row) and the current predicted values (bottom row). Substantial distributional differences are apparent. The circular correlation between the observed and corrected phase at stimulus onset (over all accepted trials) ranged over subjects: for delta, from $r=-.24$ to $r=.17$ with a mean of $r=-.03$; for theta, from $r=-.55$ to $r=.45$ with a mean of $r=-.08$; for alpha, from $r=-.57$ to $r=.78$ with a mean of $r=.29$; and for beta, from $r=-.43$ to $r=.45$ with a mean of $r=.12$. None of these means over subjects approached significance, and the coefficient of determination $\left(r^{2}\right)$ suggests that the observed and corrected phases consistently share at most (in alpha) only $8 \%$ of their variance.

\section{Discussion}

The most important outcome of this study is confirmation of the existence of preferred brain states, using an estimation procedure that avoids the data 'smearing' problem that has plagued this literature. Results from this study are both complex and extensive, with data sets from a number of EEG and ERP measures covering effects in 
each of four EEG bands. For space reasons we limit the discussion here to the relative occurrence of preferred brain states, as indicated by non-random distributions of EEG band phases at stimulus onset, and their significant effects on behaviour and ERP components. Whether these preferred brain states are functional in facilitating cortical processing is also addressed.

Across stimuli, prestimulus EEG amplitudes were generally greater in the midline; this was enhanced centrally in the theta band, and parietally in the alpha band, compatible with the expected EEG topographies (e.g., Barry et al., 2010). There were no prestimulus Go/NoGo differences in EEG, as expected from the random stimulus order. At stimulus onset there were small increases in RMS amplitudes in midline delta, particularly centrally and on the right, and in midline theta, particularly frontally and centrally. Also, small increases in alpha were notable frontally and centrally, particularly in the midline-right regions. In contrast, beta showed small frontal and left central decreases. These findings suggest the general involvement of evoked activity in response to the stimuli. The changes in RMS amplitudes reflected Go/NoGo differences that varied with band. Delta increases, particularly in the parietal midline, and central right regions, were greater for Go responses. There were no Go/NoGo differences in theta. In alpha, there was a left parietal decrease in Go and general increase in NoGo. The decrease in beta was larger for Go than NoGo, particularly in the left hemisphere and frontal midline. These effects interleave with the observed Go/NoGo ERP component differences discussed below, and the current study cannot explore whether the EEG changes facilitate the ERP differences, or whether they are simply reflections of the same phenomena.

\subsection{Artefact in previous uncorrected phase estimates}

Figure 7 illustrates, in one subject, the difference between the observed uncorrected phase estimates (as previously reported in the literature - middle row) and the current predicted values (bottom row). The differences between the two sets of distributions indicates substantial artefact. Over subjects, our correlation of the uncorrected and corrected alpha phase estimates from each subject indicated that, on average, these shared only some $8 \%$ of their variance. Other band phases had less similarity. This provides a framework for evaluating the present results in the context of the existing literature. It suggests that substantial differences will be found compared with our previous alpha study (Barry et al., 2004). Generalising this outcome beyond the alpha band to our previous narrowband studies implies that results here will bear little relation to those previous studies. In essence, this study provides novel data, and will need replication and further study to establish its validity and value.

\subsection{Preferred brain states}


The existence of preferred brain states at stimulus onset is readily apparent in the data at the individual level. For example, Figure 7 displays circular histograms of the estimated phases at stimulus onset for the Go trials of one participant, clearly demonstrating preferential phase occurrence in each band. Further, Figure 8 shows each accepted Go trial of that participant sorted on the estimated alpha phase at stimulus onset. This reinforces the nature of the non-random occurrence of phase states, and indicates how this relates to our orthogonal dimensions of phase defined in Figure 1. Figure 8 also shows how the separation of the wide-band data results in different amplitudes of the main ERP components as a function of these alpha phase dimensions. Across subjects, some of these individual effects reach significance, as reflected in the results shown in the first line of each of Tables 2 to 4. Negativity was more common in the delta band than would be expected if phase at stimulus onset occurred randomly. Negative driving was more common in delta and less common in theta and alpha. Waxing was more common than expected in delta and beta, and less common in theta and alpha. Noting that the FDR-corrected probability of a false alarm in regard to this variable is .05, the chance of 8 significant cases of preferential phase randomly occurring is $p=.05^{8}$, or $\sim 1$ in 26 billion. This finding strongly confirms the existence in this paradigm of the preferred brain states at stimulus onset.

Our most recent adult study using this paradigm, Barry et al. (2010), found that negativity occurred preferentially at 1, 2, 10 and $11 \mathrm{~Hz}$, and less often at 4 and $5 \mathrm{~Hz}$. Negative driving occurred preferentially at 2, 3, and $13 \mathrm{~Hz}$, and was reduced at 1, 6, 7, and $9 \mathrm{~Hz}$, while waxing was more common at $2 \mathrm{~Hz}$ and less common at 1 Hz. Considering the narrow bands summed in the present delta, theta and alpha bands, some parallels can be drawn between Barry et al. (2010) and the present results in delta and theta, but it is difficult to relate those results to alpha. In our previous alpha study (Barry et al., 2004) there was no difference in the occurrence of negativity vs. positivity, compatible with the present findings. Negative driving occurred significantly more often than positive driving, an effect that was not apparent here, and waxing occurred significantly more often than waning phases, an effect contrary to that found here. These differences probably reflect the substantial impact of the 'smearing' of data across time zero in our previous studies; beta was not examined previously.

Based on expectations from the random presentation of Go vs. NoGo stimuli, we predicted that these preferred phases would not differ between Go and NoGo stimuli. This prediction had been confirmed in our previous adult study (Barry et al., 2010), but our recent child study (Barry \& De Blasio, 2012) found some imbalances that were postulated to arise from the children’s late Go P3s. Our present results again indicate no adult Go/NoGo differences in the preferential occurrence of phase, supporting our hypothesis and suggesting that Barry and De Blasio’s (2012) finding was indeed probably attributable to delayed recovery of the later child P3s affecting voltages at stimulus input. 
Most importantly, the existence of preferred brain states has been confirmed here, using an estimation procedure that unequivocally avoids the data 'smearing' problem that has permeated this literature since its inception. This clear confirmation of the existence of this brain dynamics phenomenon encourages further research into its occurrence and underlying mechanism. Given the large size of these departures from random occurrence (on average, each observed preferred phase occurred some $68 \%$ more or less than individually expected), their pursuit in future studies is well-warranted. We return later to the question of whether these preferred brain states are functionally involved in behavioural and ERP responding.

\subsection{Phase effects in performance, EEG and CNV}

\subsubsection{RT}

Our previous adult study found no phase effects on RT, but there was weak evidence suggesting longer RTs in negativity phases. Here the contrary effect was apparent, with shorter RT for negativity phases in delta, but this failed to survive the FDR procedure. Together, these results appear to rule out systematic phase effects on this aspect of behavioural performance.

\subsubsection{RMS amplitudes}

Prestimulus RMS amplitudes in negativity phases were increased topographically in delta. In negative driving, prestimulus RMS amplitudes were reduced topographically in delta and theta. Waxing phases were associated with topographically-enhanced delta, alpha, and beta, and -reduced theta. Negativity phases did not affect poststimulus RMS changes. The poststimulus increase in RMS amplitude in negative driving phases was topographically larger in delta and theta. Waxing compared with waning phases produced larger poststimulus increases topographically in theta, and topographic reductions in delta and beta.

These effects are difficult to relate to the previous findings of Barry et al. (2010). For example, although the phase effects in negativity and negative driving are perhaps broadly compatible with previous findings, the prestimulus RMS enhancements in waxing phases found here differ substantially from the decreases previously noted by Barry et al. (2010) at 2, 4 and $10 \mathrm{~Hz}$. The alpha study of Barry et al. (2004) found more alpha with negativity, and no effect of negative driving, compatible with the present results. Phase effects in the RMS changes at stimulus onset are also difficult to compare. Our alpha study found poststimulus increases in negativity (similar to the present result), but a smaller increase in negative driving than positive driving, and an increase in waxing phases, two results contrary to the present data. These differences again indicate the impact of the 'smearing' of data across time zero in our previous studies. 


\subsubsection{CNV}

CNV amplitudes were larger in negativity phases in delta, but smaller in theta, alpha and beta. Increased CNVs were associated with negative driving in delta and in beta; but smaller CNVs occurred in theta and alpha. There were no effects of waxing vs. waning phases on CNV amplitudes. These phase effects in the CNV are somewhat similar to the adult effects reported in Barry et al. (2010) for negativity in the delta and theta range, and for waxing; beta was not examined. The CNV was not investigated in our previous alpha study (Barry et al., 2004).

\subsection{Phase effects in ERP components}

Overall, when considered with the phase occurrence data and the RMS data, the CNV findings confirm the substantial differences in results obtained here with the new approach to phase determination, and suggest that the present study should be considered as breaking new ground, with results that stand alone in relation to the previous literature. For that reason, the discussion of the ERP findings will not seek similarities/differences in relation to our previous results.

\subsubsection{N1-1}

Cortical negativity was associated with larger Go N1-1 amplitudes and/or frontal changes in theta and beta, and with smaller amplitudes in delta with a frontal reduction in alpha. In negative driving, amplitudes and characteristic topographic effects for Go N1-1 were enhanced in theta, and reduced in beta. Go N1-1 amplitudes were unaffected by waxing phases. Amplitudes for NoGo N1-1 in negativity were reduced in delta, and enhanced, particularly frontally, in theta. In negative driving, amplitudes and characteristic topographic effects for NoGo N11 were enhanced in theta. NoGo N1-1 amplitudes unaffected by waxing phases. Interestingly, while many of the phase effects noted were similar for Go and NoGo, there were more effects on Go N1-1 (in alpha and beta). This suggests that differential processing at this early processing stage is facilitated by phase effects in the EEG in the higher bands.

\subsubsection{PN}

Cortical negativity was associated with smaller frontal Go PN amplitudes in beta. Amplitudes for NoGo PN in negativity were reduced in delta, and frontally reduced in beta. In negative driving, amplitudes for NoGo PN were enhanced at the vertex in alpha. For waxing phases, NoGo PN amplitudes were reduced in the central right region in delta, and in the parietal midline in alpha. Most of the phase effects noted here were similar for Go and 
NoGo PN. While the frontal reduction in PN in negativity phases in beta was similar in Go and NoGo, there were four other phase effects differentially impacting the NoGo PN (c.f. the greater Go impacts on the N1-1), suggesting that EEG phase involvement in the differential Go/NoGo processing differs along the processing chain.

\subsubsection{P2}

In negative driving phases, amplitudes for Go P2 were enhanced frontally in theta, and in the right hemisphere in alpha. For waxing phases, regional Go P2 amplitudes were enhanced frontally in delta and in the left frontal region in theta. In negative driving, amplitudes for NoGo P2 were enhanced in the right central region in delta. The phase effects noted here all differ for Go and NoGo P2, suggesting that they all continue to contribute substantially to differential stimulus processing.

\subsubsection{P3}

Go P3 in negativity phases was enhanced in delta, particularly centrally and in the midline, but reduced in theta, particularly centrally. In negative driving, Go P3 was larger in beta, but smaller in theta, particularly centrally, in the midline, and at the vertex. NoGo P3 in negativity phases was larger in delta, particularly centrally, in the midline, and at the vertex; but reduced in beta in the posterior hemispheres. NoGo P3 in negative driving phases was enhanced in delta, particularly centrally, in the midline; and in beta; but reduced in theta, particularly centrally, in the midline, and at the vertex. Waxing vs. waning phases were associated with enhanced NoGo P3 in delta in the left central region. Negativity effects in delta, and negative driving effects in beta and theta are very similar for Go and NoGo, suggesting some common processing affected by EEG phase, but there are also other differential effects, presumably contributing to the differentiation of the Go/NoGo P3 into P3b/P3a.

\subsubsection{SW}

Go SW was more positive in negativity in delta in the midline, and significantly less positive in beta at the vertex. Go SW in negative driving phases was more positive in delta, particularly on the right, but more negative in theta, particularly centrally, and in the midline. NoGo SW in negativity phases were significantly more positive in delta, particularly in the midline and the central right region. NoGo SW in negative driving was more negative in theta, particularly in the central and midline regions, and less negative in beta. While there is substantial overlap between some phase effects in Go and NoGo SW, suggesting some commonality in the generation of the components, they differ in other phase effects, suggesting a continuation of phase effects to the differential Go/NoGo processing at the SW stage. 
Go LP in negativity phases was more positive in delta, particularly centrally and in the midline, and more negative in theta and alpha. Go LP in negative driving was more positive in delta, particularly centrally, and in beta, but more negative in theta, particularly centrally and in the midline. NoGo LP in negativity was more positive in delta, particularly centrally and in the midline, but less positive in theta centrally. NoGo LP in negative driving was more positive in delta, particularly in the central and midline regions, but more negative in theta, particularly in the central and midline regions. The phase effects in delta and theta are very similar for Go and NoGo LP, but there are some differences in alpha and beta that suggest ongoing phase effects even at this late stage of differential processing.

\subsection{Functionality of preferred brain states}

We have demonstrated in previous dynamics studies that the preferred brain states have functional significance. This demonstration relies on the preferred phases being associated with ERP differences that are directly related to a component's functional characteristics. We continue this exploration here. This can be examined in the phase effects on the CNV, and on each ERP component described above. If a phase $\mathrm{X}$ is associated with (say) an increase in component $\mathrm{Y}$ amplitude, and $\mathrm{X}$ is preferentially present, it readily follows that the preferential phase occurrence is linked to increases in component $\mathrm{Y}$. However, this understanding is complicated if a phase occurs significantly less often than expected by chance (indicated by a downward arrow in the first line of each Table) - in this case, the direction of the phase effect on each component must be reversed when considering its functionality. Thus, if phase $\mathrm{Z}$ is associated with an increase in component $\mathrm{Y}$ amplitude, and $\mathrm{Z}$ is preferentially absent, it follows that the preference lead to decreases in component $\mathrm{Y}$. This reversal occurs for theta and alpha bands in both negative driving (Table 3) and waxing (Table 4). We will clarify this difficulty at the first example below.

First, cortical negativity was preferentially present in the delta band (Table 2). This preferred delta phase state was associated with larger CNVs (particularly in the central midline), and smaller Go and NoGo N1-1, and NoGo PN. It was associated with enhanced NoGo P2 in the right central region, larger Go and NoGo P3s (particularly in the central midline), and with larger midline Go SWs and larger NoGo SWs (particularly in the midline and central right region). Both Go and NoGo LPs were enhanced, particularly centrally and in the midline.

Second, negative driving was preferentially present in delta, and preferentially absent in theta and alpha

(Table 3). CNV was enhanced in delta, and reduced in theta and alpha, and interpretation of these last two effects is 
the first example of the reversal required to interpret the effects. The reduction in phases that are preferentially absent in theta and alpha means that the negative driving phases are serving to enhance CNV, either directly (in delta: more phase occurrences, each associated with enhanced CNV), or indirectly (in theta and alpha: less phase occurrences, each associated with reduced CNV). So these preferred negative driving states are associated with larger CNVs in delta, theta, and alpha. We apply this reversal to all negative driving effects in theta and alpha. Thus preferred negative driving states are associated in theta with Go N1-1 reductions, particularly in the midline, posterior midline, and vertex; and NoGo N1-1 reductions, particularly in the midline and vertex. NoGo PN is preferentially reduced at the vertex in alpha. Go P2 is preferentially reduced frontally in theta and on the right in alpha; NoGo P2 is differentially reduced in the left central region in delta. Preferred negative driving states are associated with Go P3 increases, particularly in central, midline, and vertex regions in theta. NoGo P3 is preferentially enhanced in delta and theta, particularly in central and midline areas (and the vertex for theta). Preferred negative driving states are associated with Go SW increases in delta (particularly on the right) and theta (particularly in central and midline regions). NoGo SW is preferentially enhanced in theta, particularly in the central and midline regions). Go LP is enhanced in delta (particularly in the central region) and theta (particularly in central and midline regions); NoGo LP is enhanced, particularly in central and midline regions, in delta and theta preferred negative driving phases.

Third, waxing is preferentially present in delta and beta, and preferentially absent in theta and alpha (Table 4); hence theta and alpha effects are reversed in this paragraph. These states have no preferential effect on CNVs or N1-1. NoGo PN is reduced by preferred waxing phases in the right central region in delta and in the parietal midline in alpha. Go P2 is enhanced in the frontal region in delta and in the frontal right region in alpha. NoGo P3 is enhanced in the left central region by preferred waxing phases in delta.

Bringing these three sets of results together for each component shows that the CNV was enhanced by preferred negativity phase states in delta, and negative driving states in delta, theta and alpha, suggesting a close link between preferred phase states and the anticipatory preparation reflected in the CNV. Preferred phase states are associated with reduced N1-1 for both Go and NoGo, suggesting that general efficiency of N1-1 processing (indicated by smaller energy investments) is enhanced by the preferred states. NoGo PN is reduced in preferred EEG phase states, either globally or topographically, suggesting differential processing for this component. P2 is a substantial vertex positivity only for Go, suggesting completion of the identification/categorisation stage for this stimulus. Preferred waxing phases contribute to the reduced frontal and frontal right negativity characterising the Go P2 (see Figure 6), and thus facilitate this aspect of the processing chain. Preferred phase states generally contribute to larger Go and NoGo P3s, and preferential waxing phases in delta contribute to the greater left central 
activity in the NoGo P3a. There is general enhancement of both Go and NoGo SWs and LPs by preferred phase states.

These findings indicate that the preferential occurrence of particular brain states, as indicated by the nonrandom patterning of EEG phase in the delta, theta, alpha and beta bands at stimulus onset, is functionally associated with efficient stimulus processing in this paradigm. In general, CNV amplitude was increased in preferred phase states, suggesting a general increase in preparatory state. Although N1-1 was generally reduced, NoGo PN was differentially reduced in the preferred states, suggesting that differential processing was beginning to be functionally enhanced. Subsequently, P2 to Go stimuli, apparently marking completion of the Go/NoGo categorisation, was enhanced for Go stimuli presented in the preferred phase states. P3 was generally enhanced in the preferred phase states, and waxing preferentially aided the topographic shift from the Go to NoGo P3. SW and LP components were increased independently of their Go/NoGo status, indicating that the preferential phase states completed their differential contribution to the two processing chains at the P3 stage; the SW and LP may merely be the sequellae of those chains. Overall, it is clear that the preferred states are involved in the differential processing of Go and NoGo stimuli, substantially leading to some of the associated differences in ERP component amplitudes.

\subsection{Limitation}

We note here an additional limitation. As illustrated in Figure 3, phase realignments occur in the prestimulus period. In the 125 ms over which we extrapolated the uncontaminated prestimulus phase, some "phase realignments” may appear to occur because of poststimulus evoked activity "leaking” into the prestimulus period of the derived band waveform. We designed our procedure to avoid this form of artefact. However, it is also possible that genuine phase realignments may occur between -125 ms and stimulus onset. Our procedure does not capture such genuine phase shifts. It may be possible in future work to reduce this prestimulus "blind” period by reducing the duration of the FFT window, but we found in piloting our present procedure that we could not do so without degrading the FFT estimates. However, such phase resets are most likely random in time and phase, so probably cancel to some extent, in contrast to the systematic artefacts resulting from the partial inclusion of the ERP in previous phase estimates.

\subsection{Summary and future research}

We employed here for the first time a new phase estimation procedure that avoids the problem of leakage of the poststimulus data into the prestimulus epoch (i.e., across time c.f. frequency), and its contamination of the 
phase estimate. This confirmed the existence of preferred phase-defined brain states at stimulus onset in an auditory equiprobable Go/NoGo task, across the delta, theta, alpha and beta bands. We consider that these originate from the dynamic adjustment of cortical activity underlying various frequencies in the ongoing EEG. The preferred states at particular frequencies have been shown to be differentially associated with elevated prestimulus CNV amplitudes, which are probably involved in the mechanisms of some of the subsequent phase effects observed in the poststimulus ERP amplitudes. This study is the first dynamics study of this type to target PCA-derived components, allowing a substantial expansion of the range of processing stages examined here compared with previous work. Working in the context of a processing schema recently proposed for this paradigm, we have been able to show the functional contribution of our preferred phase states to the stages of processing involved.

The value of these findings can be underscored by consideration of effects on the P3, the most-studied ERP component. The preferred states generally amplified P3, a finding that would be interesting to pursue in other paradigms. Further, noting that impaired P3 is often observed in clinical samples (such as AttentionDeficit/Hyperactivity disorder; see review in Johnstone et al., 2013), this result suggests that dysfunction in this mechanism may be worth investigation in such groups. Also, preferred brain states contributed to the distinctive Go/NoGo P3 topographies (anteriorisation of the NoGo P3; Barry \& Rushby, 2006). This adds to the importance of the present results, and should encourage further investigation of the occurrence and mechanism of the preferred phase phenomenon.

We have hypothesised previously that the occurrence of cortical negativity, negative driving, and waxing at stimulus onset, in the traditional bands, is critical to cognitive functioning in terms of how they produce negativity/positivity over the cortex at particular times during the processing chain. We propose that this serves to activate/deactivate the cortex, affecting the brain regions involved in the task-related stimulus processing. Whatever the mechanism, we have confirmed in a number of studies that, in tasks involving consistently timed stimulus presentations, the phase of ongoing EEG activity is reflexively adjusted across a range of frequencies to optimise performance, extending the phase-reset model of ERP genesis. The present results confirm these effects with an artefact-free estimation of phase, and extend our understanding of the dynamic brain processes involved in perception and cognition. While the preferred phase occurrences found here would be relatively restricted to paradigms with a fixed ISI, the phase effects they produce should be widely generalisable, and hence could be usefully pursued in non-fixed ISI paradigms. 


\section{REFERENCES}

Barry, R.J., 2009. Evoked activity and EEG phase resetting in the genesis of auditory Go/NoGo ERPs. Biol. Psychol. 80, 292-299.

Barry, R.J., Clarke, A.R., 2009. Spontaneous EEG oscillations in children, adolescents, and adults: Typical development, and pathological aspects in relation to AD/HD. J. Psychophysiol. 23, 157-173.

Barry, R.J., De Blasio, F.M., 2012. EEG-ERP phase dynamics of children in the auditory Go/NoGo task. Int. J. Psychophysiol. 86, 251-261.

Barry, R.J., De Blasio, F.M., 2013. Sequential processing in the equiprobable auditory Go/NoGo task: A temporal PCA study. Int. J. Psychophysiol. 89, 123-127.

Barry, R.J., De Blasio, F.M., Borchard, J.P., (in press). Sequential processing in the equiprobable auditory Go/NoGo task: Children vs. adults. Clin. Neurophysiol.

Barry, R.J., De Blasio, F., Rushby, J.A., Clarke, A.R., 2010. Brain dynamics in the auditory Go/NoGo task as a function of EEG frequency. Int. J Psychophysiol. 78, 115-128.

Barry, R.J., de Pascalis, V., Hodder, D., Clarke, A.R., Johnstone, S.J., 2003. Preferred EEG brain states at stimulus onset in a fixed interstimulus interval auditory oddball task, and their effects on ERP components. Int. J. Psychophysiol. 47, 187-198.

Barry, R.J., Kirkakul, S., Hodder, D., 2000. EEG alpha activity and the ERP to target stimuli in an auditory oddball paradigm. Int. J. Psychophysiol. 39, 39-50.

Barry, R.J., Rushby, J.A., 2006. An orienting reflex perspective on anteriorisation of the P3 of the event-related potential. Exp. Brain. Res. 173, 539-545.

Barry, R.J., Rushby, J.A., Johnstone, S.J., Clarke, A.R., Croft, R.J., Lawrence, C.A., 2004. Event-related potentials in the auditory oddball as a function of EEG alpha phase at stimulus onset. Clin. Neurophysiol. 115, 25932601.

Barry, R.J., Rushby, J.A., Smith, J.L., Clarke, A.R., Croft, R.J., 2006. Dynamics of narrow-band EEG phase effects in the passive auditory oddball task. Eur. J. Neurosci. 24, 291-304.

Barry, R.J., Rushby, J.A., Smith, J.L., Clarke, A.R., Croft, R.J., 2009. Brain dynamics in the auditory oddball task as a function of stimulus intensity and task requirements. Int. J. Psychophysiol. 73, 313-325.

Barry, R.J., Rushby, J.A., Smith, J.L., Clarke, A.R., Croft, R.J., Wallace, M.J., 2007. Brain dynamics in the active vs. passive auditory oddball task: Exploration of narrow-band EEG phase effects. Clin. Neurophysiol. 118, 2234-2247. 
Başar, E., 1980. EEG Brain Dynamics: Relation between EEG and Brain Evoked Potentials. Elsevier/North Holland Biomedical Press, Amsterdam, 411 pp.

Başar, E., Başar-Eroglu, C., Rosen, B., Schütt, A., 1984. A new approach to endogenous event-related potentials in man: Relation between EEG and P300-wave. Int. J. Neurosci. 24, 1-21.

Başar, E., Stampfer, H.G., 1985. Important associations among EEG-dynamics, event-related potentials, short-term memory and learning. Int. J. Neurosci. 26, 161-180.

Benjamini, Y., Hochberg, Y., 1995. Controlling the false discovery rate: A practical and powerful approach to multiple testing. J. R. Statist. Soc. B 57, 289-300.

Benjamini, Y., Yekutieli, D., 2001. The control of the false discovery rate in multiple testing under dependency. Ann. Stat. 29, 1165-1188.

Berens, P., 2009. CircStat: A MATLAB toolbox for circular statistics. J. Stat. Softw. 31, 1-21.

Callaway, E., Yeager, C.L., 1960. Relationship between reaction time and electroencephalographic alpha phase. Science 132, 1765-1766.

Croft, R.J., Barry, R.J., 2000. EOG correction of blinks with saccade coefficients: A test and revision of the aligned-artifact average solution. Clin. Neurophysiol. 111, 444-451.

Delorme, A., Makeig, S., 2004. EEGLAB: An open source toolbox for analysis of single-trial EEG dynamics including independent component analysis. J. Neurosci. Methods 134, 9-21.

Dien, J., 2010. The ERP PCA Toolkit: An open source program for advanced statistical analysis of event-related potential data. J. Neurosci. Methods 187, 138-145.

Howell, D.C., 1997. Statistical Methods for Psychology, 4th edn., Duxbury Press, California.

Jansen, B.H., Brandt, M.E., 1991. The effect of the phase of prestimulus alpha activity on the averaged visual evoked response. Electroenceph. Clin. Neurophysiol. 80, 241-250.

Johnstone, S.J., Barry, R.J., Clarke, A.R., 2013. Ten years on: A follow-up review of ERP research in attentiondeficit/hyperactivity disorder. Clin. Neurophysiol. 124, 644-657.

Johnstone, S.J., Pleffer, C.B., Barry, R.J., Clarke, A.R., Smith, J.L., 2005. Development of inhibitory processing during the Go/NoGo task: A behavioural and event-related potential study of children and adults. J. Psychophysiol. 19, 11-23.

Kayser, J., Tenke, C.E., 2003. Optimizing PCA methodology for ERP component identification and measurement: Theoretical rationale and empirical evaluation. Clin. Neurophysiol. 114, 2307-2325.

Lorenzo-Seva, U., ten Berge, J.M.F., 2006. Tucker's congruence coefficient as a meaningful index of factor similarity. Methodology 2, 57-64. 
Makeig, S., Delorme, A., Westerfield, M., Jung, T.P., Townsend, J., Courchesne, E., Sejnowski, T.J., 2004.

Electroencephalographic brain dynamics following manually responded visual targets. PLoS Biol. 2, 747762.

O’Brien, R.G., Kaiser, M.K., 1985. MANOVA method for analyzing repeated measures designs: An extensive primer. Psychol. Bull. 97, 316-333.

Pleydell-Pearce, C.W., 1994. DC potential correlates of attention and cognitive load. Cogn. Neuropsychol. 11, 149166.

Rémond, A., Lesèvre, N., 1967. Variations in average visual evoked potentials as a function of the alpha rhythm phase (“autostimulation”). Electroenceph. Clin. Neurophysiol. Suppl. 26, 42-52.

Rockstroh, B., Elbert, T., Canavan, A., Lutzenberger, W., Birbaumer. N., 1989. Slow Cortical Potentials and Behaviour, 2nd edn., Urban and Schwarzenberg, Munich, 267 pp.

Tabachnick, B.G., Fidell, L.S., 1989. Using Multivariate Statistics, 2nd edn., Harper Collins, N.Y, 746 pp.

Trimble, J.L., Potts, A.M., 1975. Ongoing occipital rhythms and the VER. I. Stimulation at peaks of the alpharhythm. Investig. Opthamol. Vis. Sci. 14, 537-546.

Tucker, L.R., 1951. A method for synthesis of factor analysis studies, Personnel Research Section Report No. 984, Department of the Army, Washington, D.C. 


\section{Figure Legends}

Figure 1. Left: A schematic representation of the narrow-band EEG phase definitions. Phases $(A+B)$ define cortical negativity, $(\mathrm{A}+\mathrm{D})$ define negative driving, and $(\mathrm{A}+\mathrm{C})$ define waxing. For each pair defining a dimension (e.g., A + D), the remaining two phases (e.g., B + C) define the other extreme of that dimension. These three orthogonal dimensions are easier to conceptualise than traditional phase measures (degrees or radians). Right: The circular motion form of this schema.

Figure 2. A schematic showing the major steps in EEG processing, emphasising the novel aspects of the phase determinations and their use in separating ERP components for analysis. Note: GM = Grand Mean.

Figure 3. Illustration of the processing approach using an arbitrarily-selected Go trial (number 32) from an arbitrarily-selected subject (S08). Data are shown for the $4 \mathrm{~Hz}$ and $6 \mathrm{~Hz}$ narrow-band frequencies forming the theta band. For the first two columns, the data show the magnitude and phase at each millisecond from -500 to $+500 \mathrm{~ms}$ around stimulus onset, derived from 1001 FFTs. The third row shows the instantaneous amplitude derived from the magnitude and phase at each point. Time 0 is stimulus onset. The dashed vertical line marks the last data point uncontaminated by poststimulus data $(-125 \mathrm{~ms})$. Four phase resets are circled in red (colour is shown on the web version) on the $4 \mathrm{~Hz}$ phase plot. The bottom row shows the observed phase at $-125 \mathrm{~ms}$ from the second row (dashed line to unfilled mark on the unit circle); the extrapolated phase at stimulus onset is indicated by the black line to the black mark. These 4 and $6 \mathrm{~Hz}$ phase and magnitude data were combined using vector addition to form the theta phase and magnitude plots, which yielded the theta amplitude plot. The estimated theta phase at stimulus onset was obtained from the 4 and $6 \mathrm{~Hz}$ estimated values, again using vector addition.

Figure 4. A: Prestimulus RMS amplitudes are shown as a function of frequency at each analysed site; bands are indicated at Pz. There are no substantial Go/NoGo differences; lines essentially overlap. B: Change from prestimulus to poststimulus RMS amplitudes; here, Go/NoGo differences vary with frequency and site.

Figure 5. Left: Grand mean ERPs for each condition are shown at the midline sites. Note the large Go/NoGo difference in the P3. The Go response (black line) shows a markedly posterior dominance; the NoGo response (grey line) is dominant at Cz. Right: Reconstituted ERPs from the sum of the 8 most dominant PCA factors. A good fit with the raw ERPs is apparent. 
Figure 6: The top row shows the topographic headmaps of the first eight PCA components. Below these is shown the factor order, the proposed component label, the \% variance carried by each component, and its latency. The middle plot shows the scaled factor loadings, with the factors labelled in order of their importance in the rotated output. The headmaps below indicate the component topography for Go and NoGo, and these can be compared with the raw ERP components identified in the midline plots of Figure 4.

Figure 7. Distribution of phase at stimulus onset in each band for the Go trials of a single subject (S08). These circular histograms show the number of trials with phase in each $\pi / 10$ interval. The radial scale varies with the concentration of preferred phase in order to better display the data; the scale is indicated by the grey numbers near $\pi / 2$. Top row: observed phase at $-125 \mathrm{~ms}$; middle row: observed phase at stimulus onset; bottom row: estimated phase at stimulus onset.

Figure 8. Example of the occurrence of preferred brain states at stimulus onset in Go data from a single subject (S08). Panel a shows raw EEG at $\mathrm{Cz}$ for all accepted Go trials in order of stimulus presentation, with one horizontal line per trial showing the amplitude at each time point; a black dot marks the RT on that trial. The mean across trials is shown below as the traditional ERP, with a dashed vertical line indicating the mean RT. Panel b shows the corresponding alpha data stream sorted by the estimated/predicted phase at stimulus onset, with the four phases defined in Figure 1 indicated. Panel c shows the mean alpha-band ERP from each phase. Panel d shows the raw trials from Panel a rearranged in the phase order of Panel b. The mean ERP from each phase division is shown in Panel e. Note in panels c and e that there is only a slight alpha phase effect on mean RT. 
Table 1. Statistics for the grand mean CNV and each analysed component identified in the grand mean PCA, in latency order.

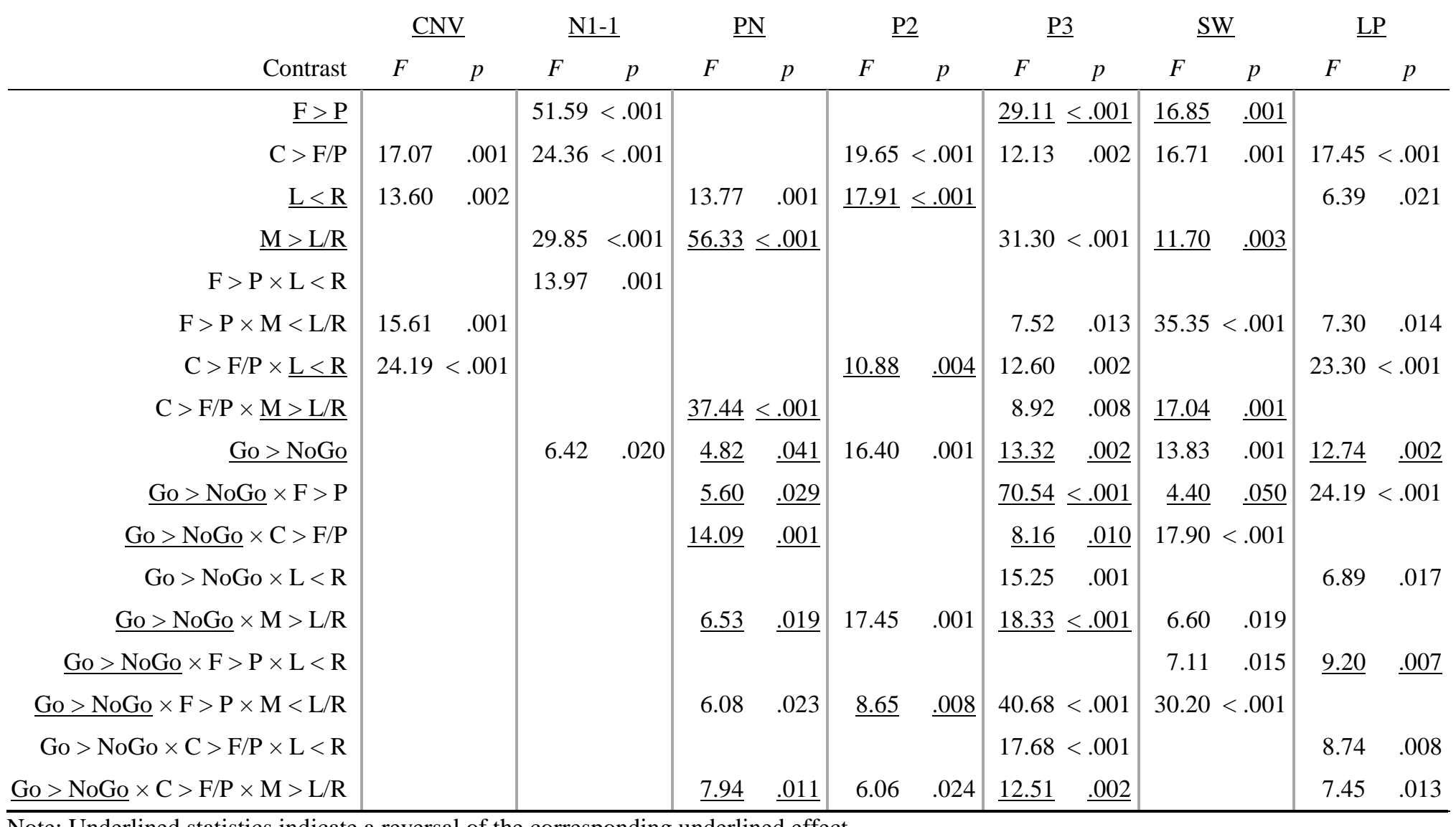


Table 2. Significant phase effects obtained in the dependent variables as a function of negativity vs. positivity.

\begin{tabular}{|c|c|c|c|c|c|c|}
\hline \multirow{2}{*}{\multicolumn{2}{|c|}{ Dependent Variable }} & \multirow{2}{*}{ Effect } & \multicolumn{4}{|c|}{ Frequency Band } \\
\hline & & & Delta & Theta & Alpha & Beta \\
\hline Number & & phase & $\uparrow * * *$ & & & \\
\hline Prestimulus & & phase $\times \mathrm{L}<\mathrm{R}$ & $\uparrow * * *$ & & & \\
\hline RMS amplitude & & phase $\times \mathrm{C}>\mathrm{F} / \mathrm{P} \times \mathrm{L}<\mathrm{R}$ & $\uparrow * * *$ & & & \\
\hline CNV amplitude & & phase & $\uparrow * * *$ & $\downarrow * * *$ & $\downarrow * * *$ & $\downarrow * * *$ \\
\hline & & phase $\times \mathrm{C}>\mathrm{F} / \mathrm{P}$ & $\uparrow * * *$ & $\downarrow * * *$ & & $\downarrow * *$ \\
\hline & & phase $\times M>L / R$ & $\uparrow * * *$ & $\downarrow * * *$ & $\downarrow^{*}$ & $\downarrow^{* *}$ \\
\hline N1 amplitude & Go & phase & $\downarrow * * *$ & $\uparrow^{*}$ & & \\
\hline & & phase $\times \mathrm{F}>\mathrm{P}$ & & $\uparrow *$ & $\downarrow^{*}$ & $\uparrow^{*}$ \\
\hline & NoGo & phase & $\downarrow * * *$ & $\uparrow^{*}$ & & \\
\hline & & phase $\times \mathrm{F}>\mathrm{P}$ & & $\uparrow^{*}$ & & \\
\hline PN amplitude & Go & phase $\times \mathrm{F}>\mathrm{P}$ & & & & $\downarrow * * *$ \\
\hline & NoGo & phase & $\downarrow * *$ & & & \\
\hline & & phase $\times \mathrm{F}<\mathrm{P}$ & & & & $\downarrow * *$ \\
\hline P3 amplitude & Go & phase & $\uparrow * * *$ & $\downarrow^{* *}$ & & \\
\hline & & phase $\times \mathrm{C}>\mathrm{F} / \mathrm{P}$ & $\uparrow * *$ & $\downarrow *$ & & \\
\hline & & phase $\times M>L / R$ & $\uparrow * * *$ & & & \\
\hline & NoGo & phase & $\uparrow * * *$ & & & \\
\hline & & phase $\times \mathrm{C}>\mathrm{F} / \mathrm{P}$ & $\uparrow * *$ & & & \\
\hline & & phase $\times M>L / R$ & $\uparrow * * *$ & & & \\
\hline & & phase $\times \mathrm{F}<\mathrm{P} \times \mathrm{M}<\mathrm{L} / \mathrm{R}$ & & & & $\downarrow * *$ \\
\hline & & phase $\times \mathrm{C}>\mathrm{F} / \mathrm{P} \times \mathrm{M}>\mathrm{L} / \mathrm{R}$ & $\uparrow * *$ & & & \\
\hline SW positivity & Go & phase $\times \mathrm{M}>\mathrm{L} / \mathrm{R}$ & $\uparrow * * *$ & & & \\
\hline & & phase $\times \mathrm{C}>\mathrm{F} / \mathrm{P} \times \mathrm{M}>\mathrm{L} / \mathrm{R}$ & & & & $\downarrow^{*}$ \\
\hline & NoGo & phase & $\uparrow * * *$ & & & \\
\hline & & phase $\times \mathrm{M}>\mathrm{L} / \mathrm{R}$ & $\uparrow * * *$ & & & \\
\hline & & phase $\times \mathrm{C}>\mathrm{F} / \mathrm{P} \times \mathrm{L}<\mathrm{R}$ & $\uparrow * * *$ & & & \\
\hline LP amplitude & Go & phase & $\uparrow * * *$ & $\downarrow^{*}$ & $\downarrow^{*}$ & \\
\hline & & phase $\times \mathrm{C}>\mathrm{F} / \mathrm{P}$ & $\uparrow * * *$ & & & \\
\hline & & phase $\times M>L / R$ & $\uparrow * * *$ & & & \\
\hline & NoGo & phase & $\uparrow * * *$ & & & \\
\hline & & phase $\times \mathrm{C}>\mathrm{F} / \mathrm{P}$ & $\uparrow * * *$ & $\downarrow^{*}$ & & \\
\hline & & phase $\times \mathrm{M}>\mathrm{L} / \mathrm{R}$ & $\uparrow * * *$ & & & \\
\hline
\end{tabular}

$\uparrow$ larger for negativity; $\downarrow$ smaller for negativity; *significant at $\mathrm{p}<.05$; ** significant at $\mathrm{p}<.01$; $* * *$ significant at $\mathrm{p}<.005$. 
Table 3. Significant phase effects obtained in the dependent variables as a function of negative driving vs. positive driving.

\begin{tabular}{|c|c|c|c|c|c|c|}
\hline \multirow{2}{*}{\multicolumn{2}{|c|}{ Dependent Variable }} & \multirow{2}{*}{ Effect } & \multicolumn{4}{|c|}{ Frequency Band } \\
\hline & & & \multirow{2}{*}{$\frac{\text { Delta }}{\text { 个*** }}$} & \multirow{2}{*}{$\begin{array}{l}\text { Theta } \\
\downarrow \downarrow * *\end{array}$} & \multirow{2}{*}{$\begin{array}{c}\text { Alpha } \\
\downarrow^{* * *}\end{array}$} & \multirow{2}{*}{$\begin{array}{c}\text { Beta } \\
\downarrow \downarrow .059\end{array}$} \\
\hline Number & & phase & & & & \\
\hline Prestimulus & & phase $\times \mathrm{C}>\mathrm{F} / \mathrm{P}$ & $\downarrow * * *$ & $\downarrow * * *$ & & \\
\hline RMS amplitude & & phase $\times \mathrm{C}>\mathrm{F} / \mathrm{P} \times \mathrm{L}<\mathrm{R}$ & & $\downarrow * * *$ & & \\
\hline Poststimulus & & phase $\times \mathrm{C}>\mathrm{F} / \mathrm{P}$ & $\uparrow^{*}$ & $\uparrow^{* *}$ & & \\
\hline Increase in & & phase $\times \mathrm{C}>\mathrm{F} / \mathrm{P} \times \mathrm{M}>\mathrm{L} / \mathrm{R}$ & & $\uparrow * * *$ & & $\downarrow^{*}$ \\
\hline \multicolumn{7}{|l|}{ RMS amplitude } \\
\hline \multirow{5}{*}{\multicolumn{2}{|c|}{ CNV amplitude }} & phase & $\uparrow * * *$ & $\downarrow * * *$ & $\downarrow * * *$ & $\uparrow * * *$ \\
\hline & & phase $\times \mathrm{F}<\mathrm{P}$ & & & $\downarrow * *$ & \\
\hline & & phase $\times \mathrm{C}>\mathrm{F} / \mathrm{P}$ & $\uparrow * * *$ & $\downarrow * * *$ & $\downarrow * * *$ & $\uparrow * * *$ \\
\hline & & phase $\times \mathrm{M}>\mathrm{L} / \mathrm{R}$ & $\uparrow * * *$ & $\downarrow * * *$ & $\downarrow * * *$ & $\uparrow * * *$ \\
\hline & & phase $\times \mathrm{C}>\mathrm{F} / \mathrm{P} \times \mathrm{M}>\mathrm{L} / \mathrm{R}$ & $\uparrow * *$ & $\downarrow^{* *}$ & & \\
\hline \multirow[t]{8}{*}{ N1 amplitude } & Go & phase & & $\uparrow * * *$ & & $\downarrow *$ \\
\hline & & phase $\times \mathrm{F}<\mathrm{P}$ & & & & $\downarrow * * *$ \\
\hline & & phase $\times M>L / R$ & & $\uparrow * * *$ & & \\
\hline & & phase $\times \mathrm{F}<\mathrm{P} \times \mathrm{M}>\mathrm{L} / \mathrm{R}$ & & $\uparrow *$ & & \\
\hline & & phase $\times \mathrm{C}>\mathrm{F} / \mathrm{P} \times \mathrm{M}>\mathrm{L} / \mathrm{R}$ & & $\uparrow * * *$ & & \\
\hline & NoGo & phase & & $\uparrow * * *$ & & \\
\hline & & phase $\times \mathrm{M}>\mathrm{L} / \mathrm{R}$ & & $\uparrow * * *$ & & \\
\hline & & phase $\times \mathrm{C}>\mathrm{F} / \mathrm{P} \times \mathrm{M}>\mathrm{L} / \mathrm{R}$ & & $\uparrow^{*}$ & & \\
\hline PN amplitude & NoGo & phase $\times \mathrm{C}>\mathrm{F} / \mathrm{P} \times \mathrm{M}>\mathrm{L} / \mathrm{R}$ & & & $\uparrow *$ & \\
\hline \multirow[t]{3}{*}{ P2 amplitude } & Go & phase $\times \mathrm{F}>\mathrm{P}$ & & $\uparrow *$ & & \\
\hline & & phase $\times \mathrm{L}<\mathrm{R}$ & & & $\uparrow * *$ & \\
\hline & NoGo & phase $\times \mathrm{C}>\mathrm{F} / \mathrm{P} \times \mathrm{L}<\mathrm{R}$ & & $\uparrow * *$ & & \\
\hline \multirow[t]{8}{*}{ P3 amplitude } & Go & phase & & $\downarrow * * *$ & & $\uparrow * * *$ \\
\hline & & phase $\times \mathrm{C}>\mathrm{F} / \mathrm{P}$ & & $\downarrow * * *$ & & \\
\hline & & phase $\times \mathrm{M}>\mathrm{L} / \mathrm{R}$ & & $\downarrow * * *$ & & \\
\hline & & phase $\times \mathrm{C}>\mathrm{F} / \mathrm{P} \times \mathrm{M}>\mathrm{L} / \mathrm{R}$ & & $\downarrow * * *$ & & \\
\hline & NoGo & phase & $\uparrow * * *$ & $\downarrow * * *$ & & $\uparrow * *$ \\
\hline & & phase $\times \mathrm{C}>\mathrm{F} / \mathrm{P}$ & $\uparrow * *$ & $\downarrow * *$ & & \\
\hline & & phase $\times \mathrm{M}>\mathrm{L} / \mathrm{R}$ & $\uparrow * * *$ & $\downarrow * * *$ & & \\
\hline & & phase $\times \mathrm{C}>\mathrm{F} / \mathrm{P} \times \mathrm{M}>\mathrm{L} / \mathrm{R}$ & & $\downarrow * *$ & & \\
\hline \multirow[t]{7}{*}{ SW positivity } & Go & phase & $\uparrow * * *$ & $\downarrow * * *$ & & \\
\hline & & phase $\times \mathrm{C}>\mathrm{F} / \mathrm{P}$ & & $\downarrow * * *$ & & \\
\hline & & phase $\times \mathrm{L}<\mathrm{R}$ & $\downarrow * * *$ & & & \\
\hline & & phase $\times M>L / R$ & & $\downarrow * * *$ & & \\
\hline & NoGo & phase & & $\downarrow * * *$ & & $\uparrow * *$ \\
\hline & & phase $\times \mathrm{C}>\mathrm{F} / \mathrm{P}$ & & $\downarrow^{*}$ & & \\
\hline & & phase $\times M>L / R$ & & $\downarrow * * *$ & & \\
\hline \multirow[t]{6}{*}{ LP amplitude } & Go & phase & $\uparrow * * *$ & $\downarrow * * *$ & & $\downarrow * * *$ \\
\hline & & phase $\times C>F / P$ & $\uparrow * *$ & $\downarrow * * *$ & & \\
\hline & & phase $\times M>L / R$ & & $\downarrow * * *$ & & \\
\hline & NoGo & phase & $\uparrow * * *$ & $\downarrow * * *$ & & \\
\hline & & phase $\times \mathrm{C}>\mathrm{F} / \mathrm{P}$ & $\uparrow * * *$ & $\downarrow * * *$ & & \\
\hline & & phase $\times M>L / R$ & $\uparrow * * *$ & $\downarrow * * *$ & & \\
\hline
\end{tabular}

$\uparrow$ larger for negative driving; $\downarrow$ smaller for negative driving; *significant at $\mathrm{p}<.05$; **significant at $\mathrm{p}$ $<.01$; ***significant at $\mathrm{p}<.005$; probability is indicated for effects approaching significance. 
Table 4. Significant phase effects obtained in the dependent variables as a function of waxing vs. waning.

\begin{tabular}{|c|c|c|c|c|c|}
\hline \multirow{2}{*}{ Dependent Variable } & \multirow{2}{*}{ Effect } & \multicolumn{4}{|c|}{ Frequency Band } \\
\hline & & Delta & Theta & Alpha & Beta \\
\hline Number & phase & $\uparrow * * *$ & $\downarrow * * *$ & $\downarrow * * *$ & $\uparrow * * *$ \\
\hline Prestimulus & phase & $\uparrow * * *$ & $\downarrow^{*}$ & $\uparrow *$ & \\
\hline \multirow[t]{3}{*}{ RMS amplitude } & phase $\times \mathrm{C}>\mathrm{F} / \mathrm{P}$ & $\uparrow * * *$ & $\downarrow * * *$ & & \\
\hline & phase $\times M>L / R$ & $\uparrow * * *$ & $\downarrow * * *$ & $\uparrow * * *$ & $\uparrow *$ \\
\hline & phase $\times \mathrm{C}>\mathrm{F} / \mathrm{P} \times \mathrm{M}>\mathrm{L} / \mathrm{R}$ & $\uparrow * *$ & $\downarrow * *$ & & \\
\hline Poststimulus & phase $\times \mathrm{C}>\mathrm{F} / \mathrm{P}$ & & $\uparrow^{*}$ & & \\
\hline Increase in & phase $\times \mathrm{M}>\mathrm{L} / \mathrm{R}$ & $\downarrow^{*}$ & $\uparrow * * *$ & & $\downarrow^{*}$ \\
\hline RMS amplitude & phase $\times \mathrm{M}>\mathrm{L} / \mathrm{R} \times \mathrm{Go}>$ NoGo & & & & $\downarrow * *$ \\
\hline \multirow[t]{2}{*}{ PN amplitude } & phase $\times \mathrm{C}>\mathrm{F} / \mathrm{P} \times \mathrm{L}<\mathrm{R}$ & $\downarrow^{* *}$ & & & \\
\hline & phase $\times \mathrm{F}<\mathrm{P} \times \mathrm{M}>\mathrm{L} / \mathrm{R}$ & & & $\downarrow^{*}$ & \\
\hline \multirow[t]{2}{*}{ P2 amplitude } & phase $\times F>P$ & $\uparrow *$ & & & \\
\hline & phase $\times \mathrm{F}>\mathrm{P} \times \mathrm{L}>\mathrm{R}$ & & $\uparrow * * *$ & & \\
\hline P3 amplitude & phase $\times \mathrm{C}>\mathrm{F} / \mathrm{P} \times \mathrm{L}>\mathrm{R}$ & $\uparrow^{*}$ & & & \\
\hline
\end{tabular}

$\uparrow$ larger for waxing; $\downarrow$ smaller for waxing; *significant at $\mathrm{p}<.05$; ** significant at $\mathrm{p}<.01$; ***significant at $\mathrm{p}<.005$. 
Figure 1.

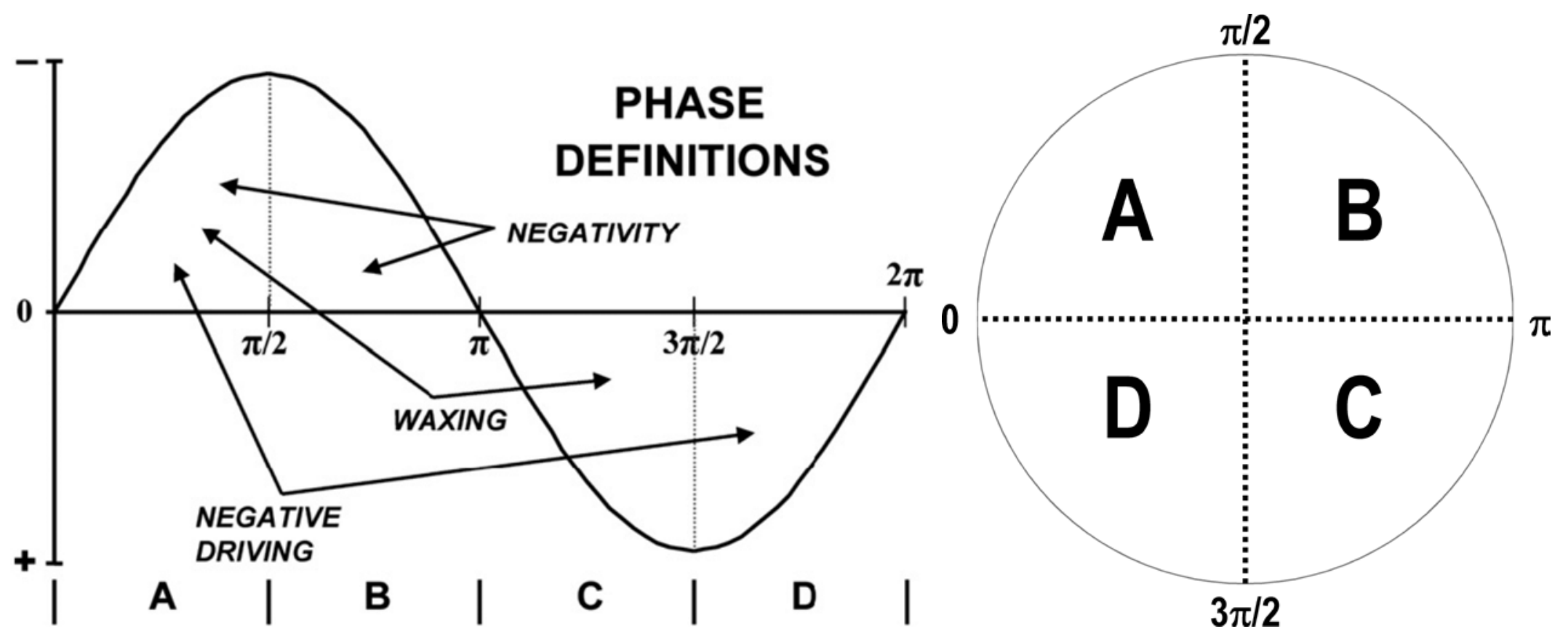


Figure 2.

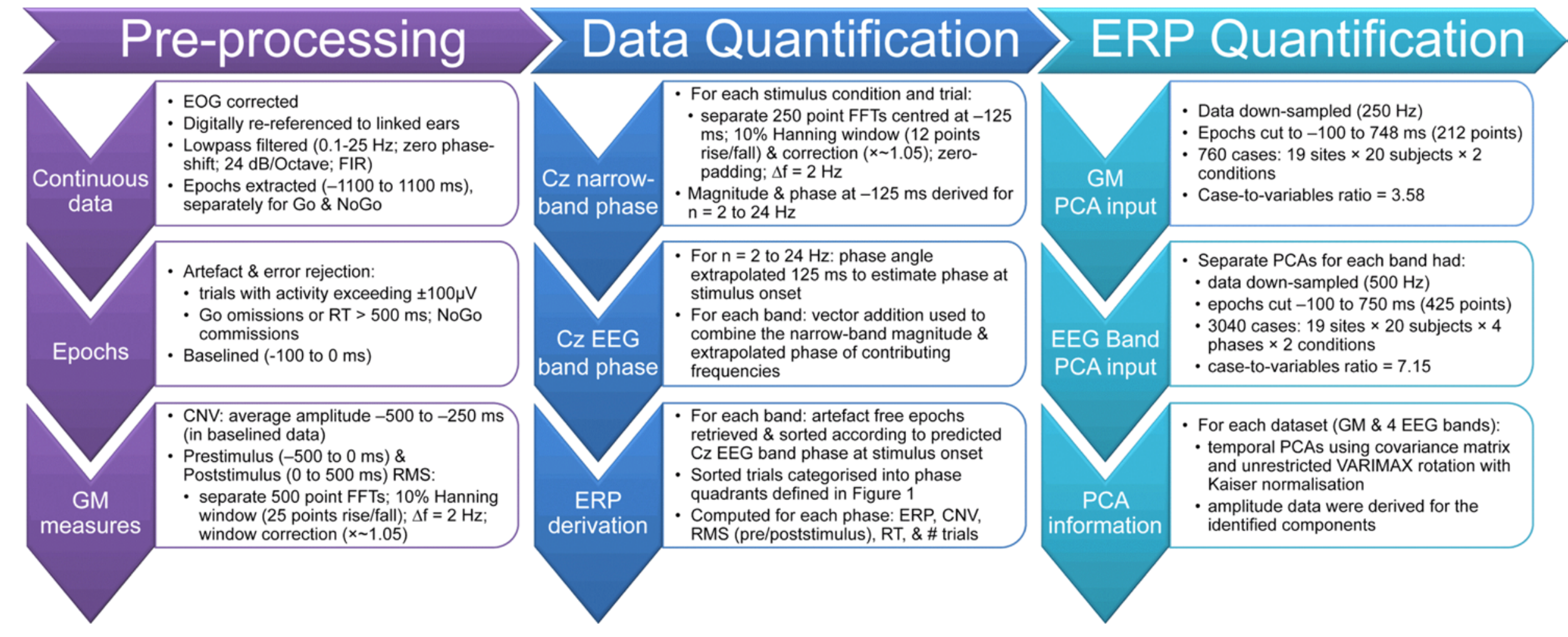


Figure 3.
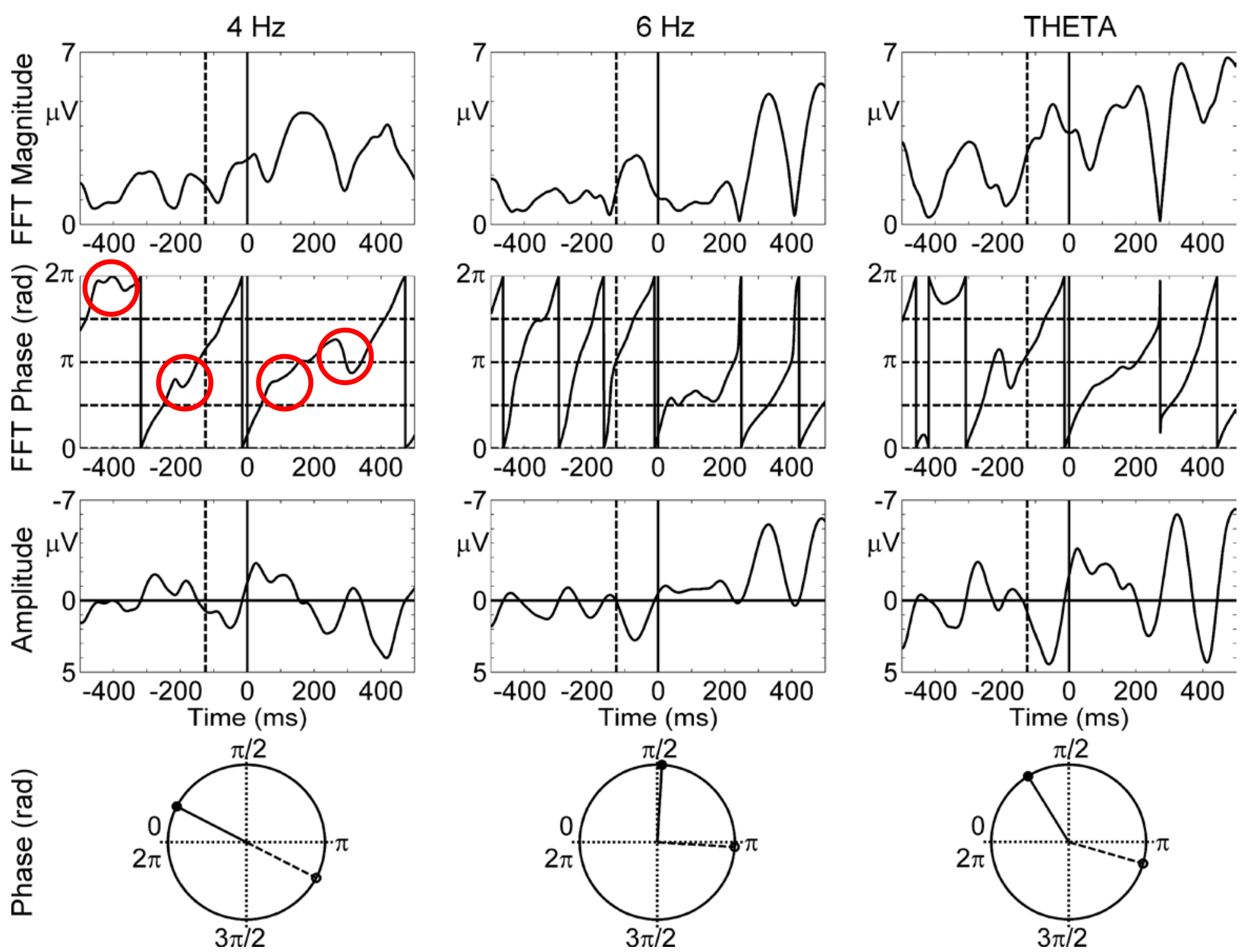
Figure 4.
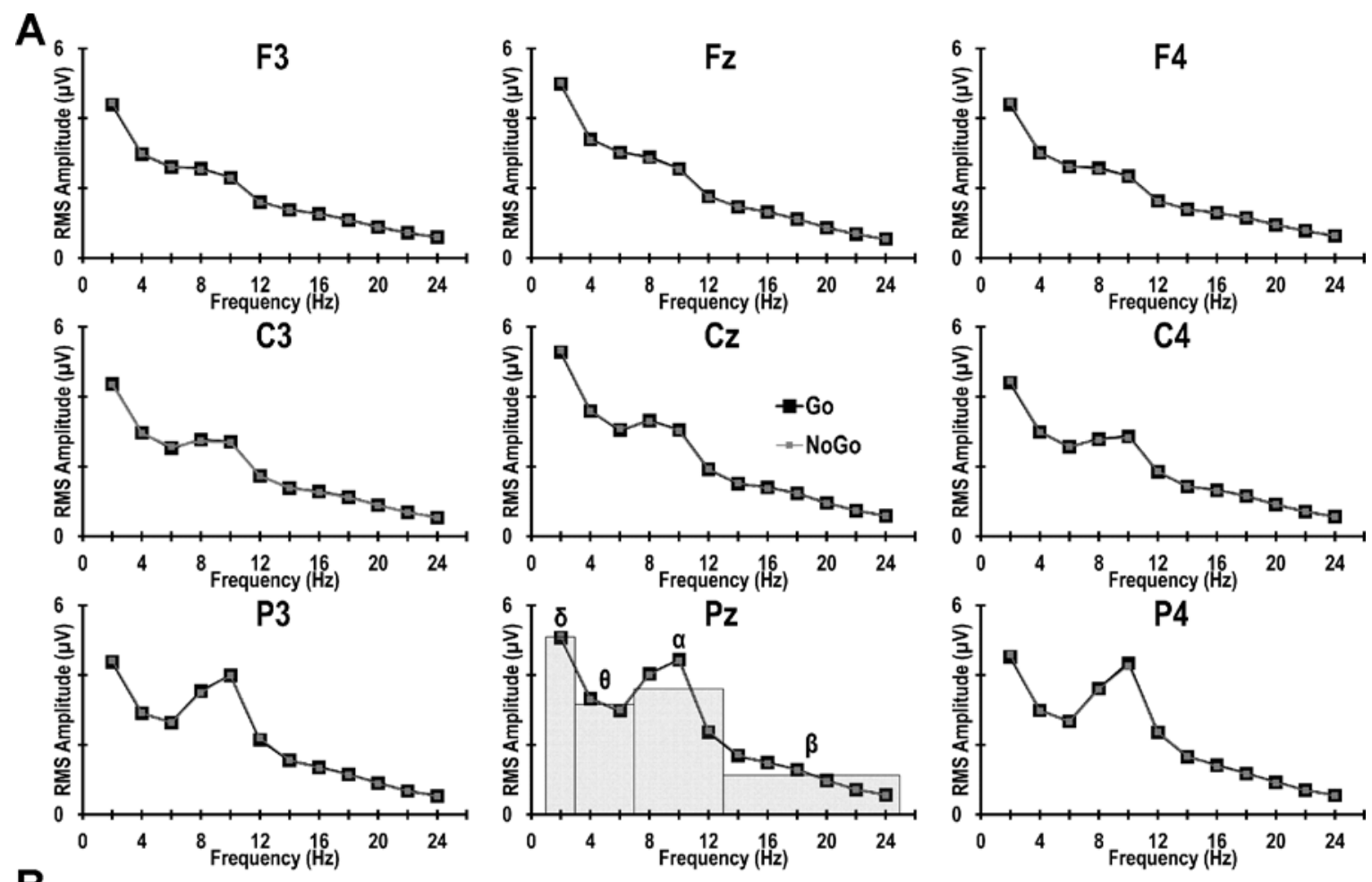

B
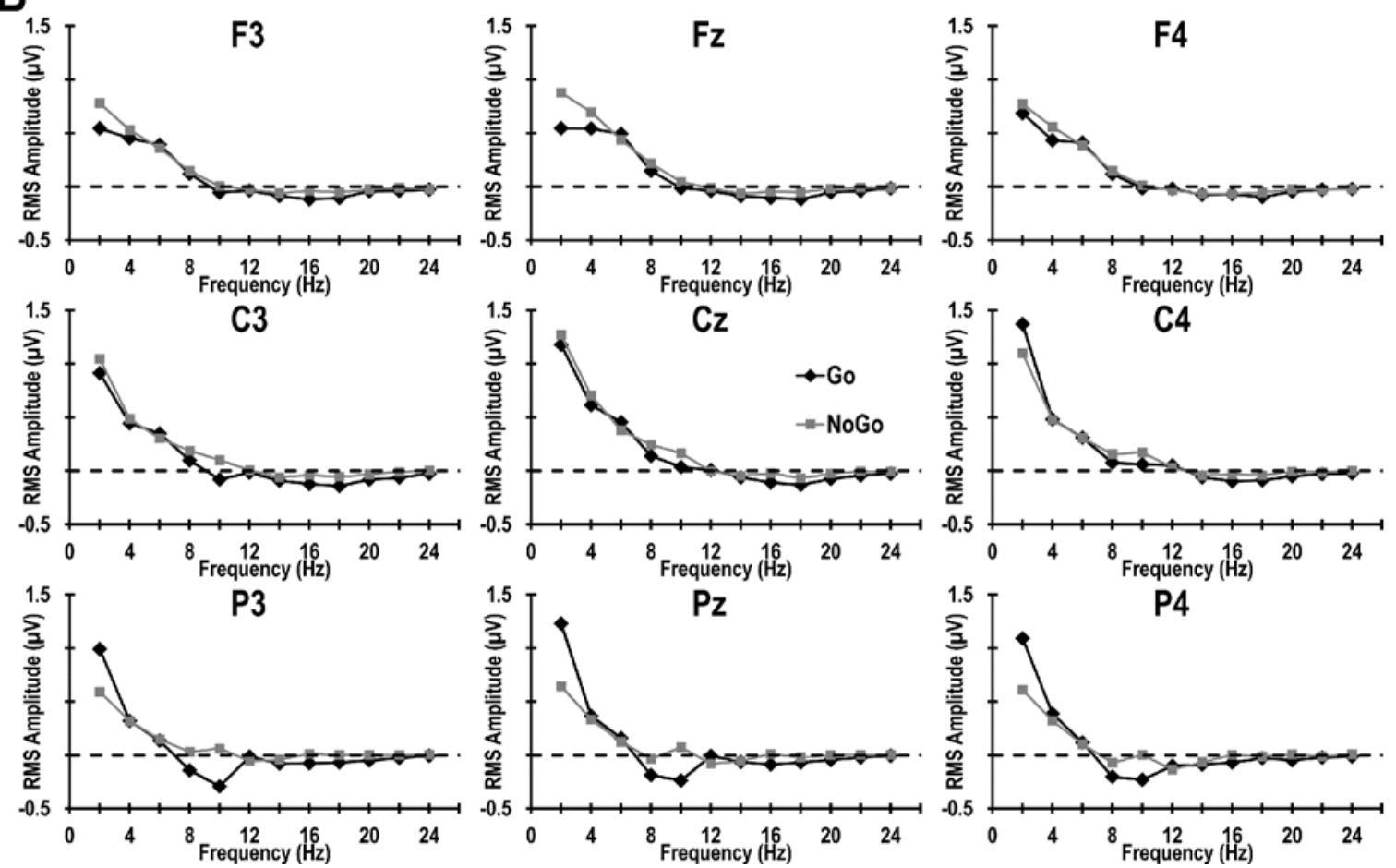
Figure 5.
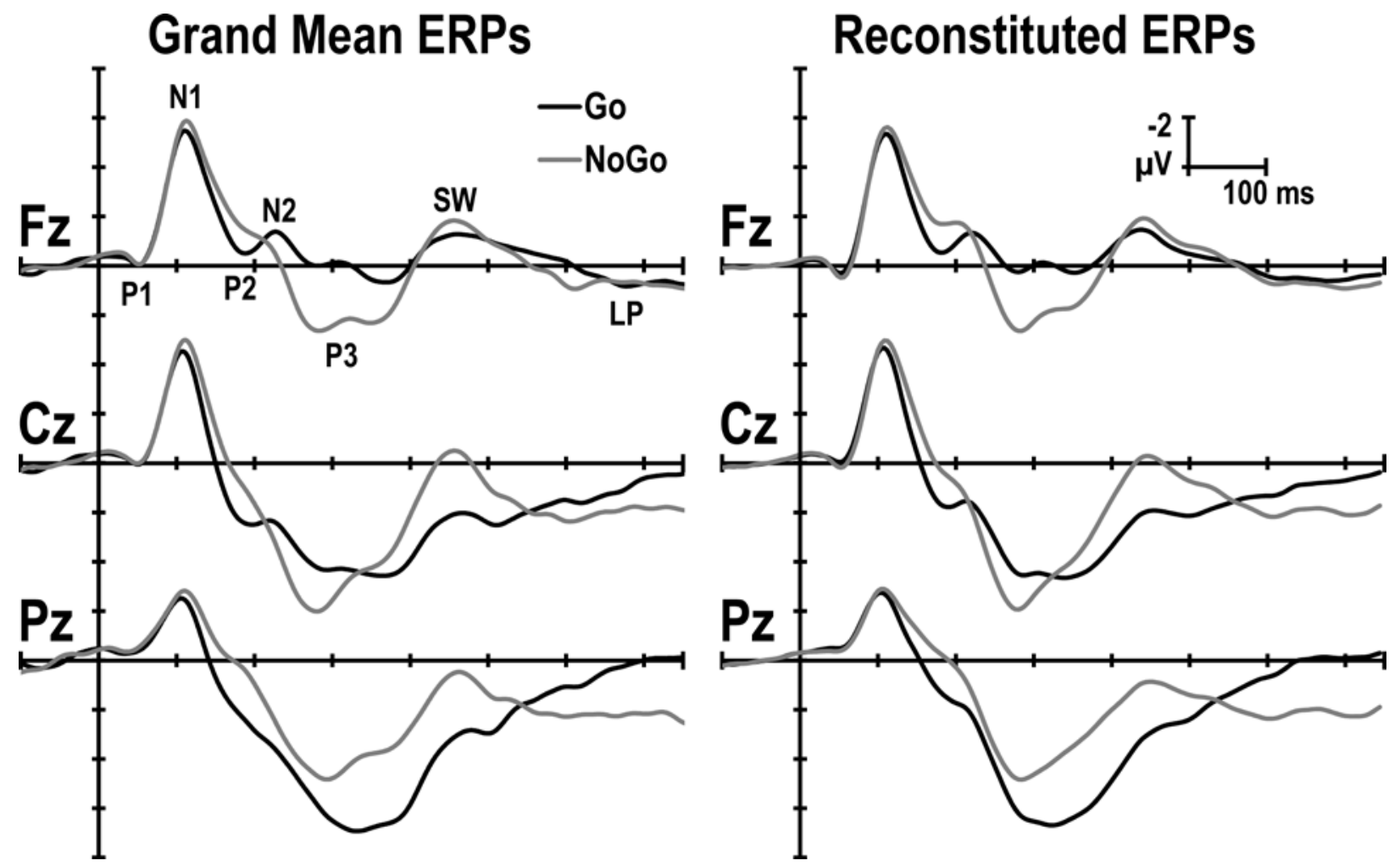

Figure 6.
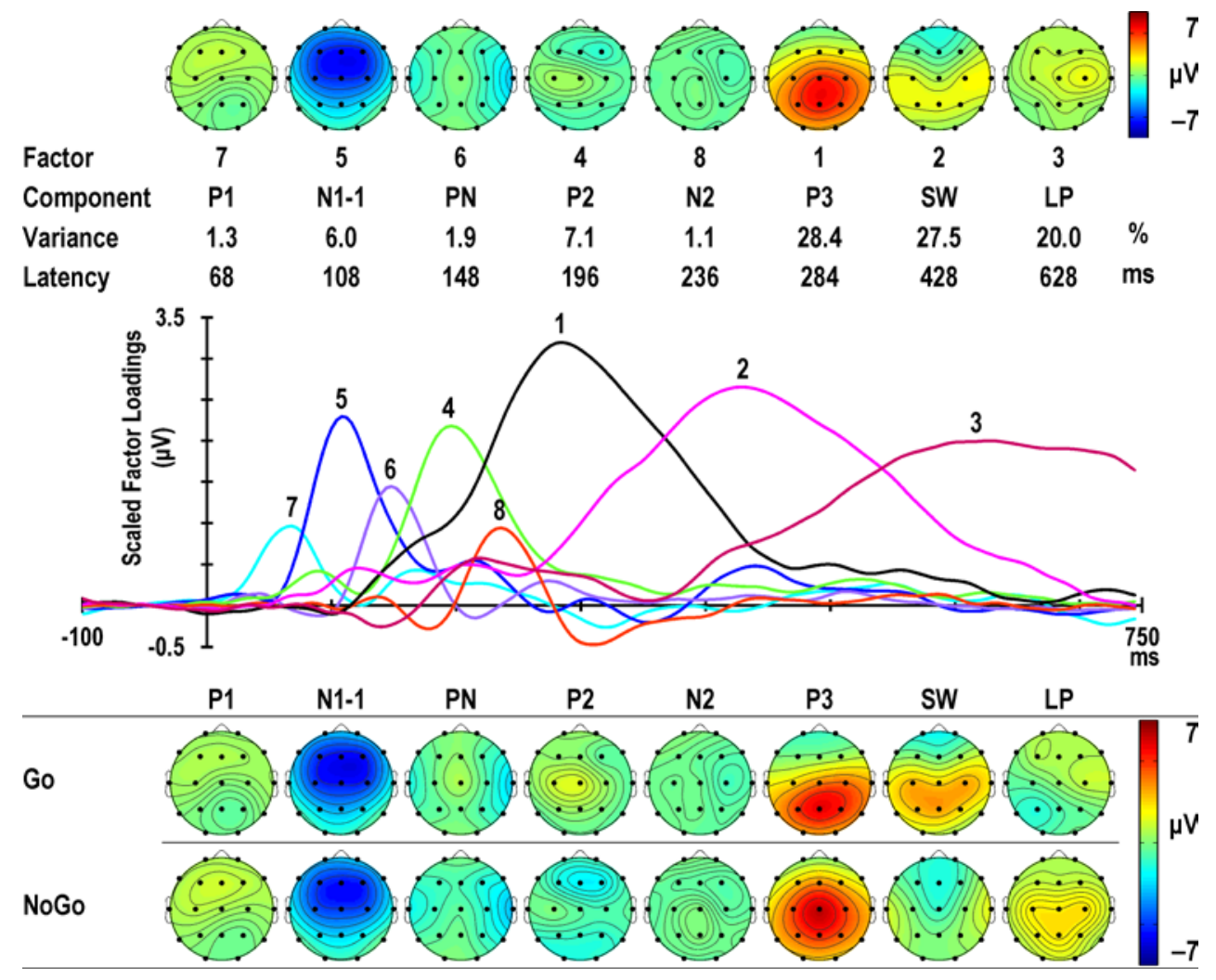
Figure 7.

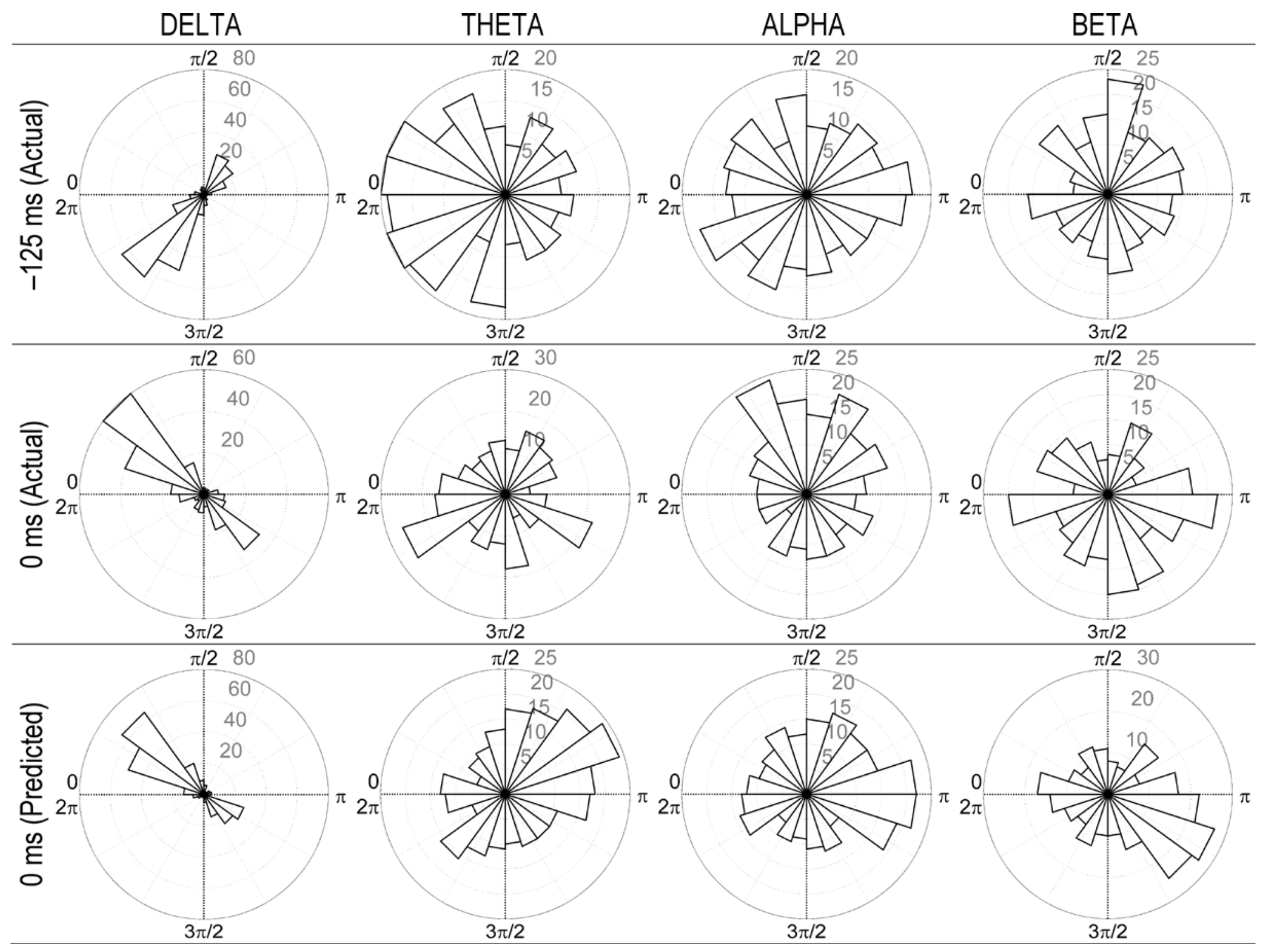


Figure 8.

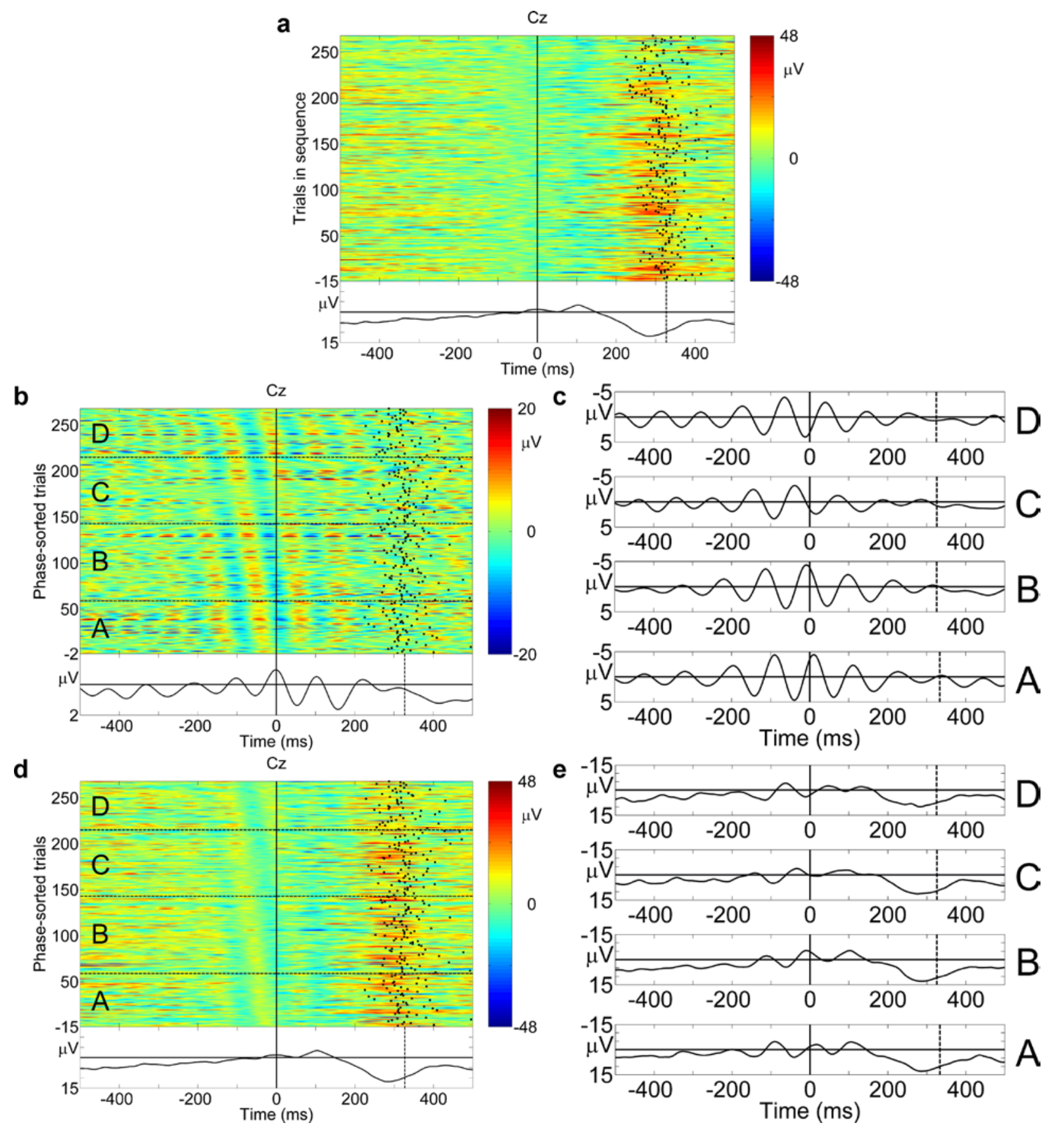

